\title{
Multiple finger propagation modes in Hele-Shaw channels of variable depth
}

\author{
Alice B. Thompson $\dagger$, Anne Juel and Andrew L. Hazel \\ School of Mathematics, University of Manchester, Oxford Road, Manchester M13 9PL, UK
}

(Received 24 June 2013; revised 12 December 2013; accepted 19 February 2014; first published online 28 March 2014)

We consider the propagation of an air finger into a wide fluid-filled channel with a spatially varying depth profile. Our aim is to understand the origin of the multiple coexisting families of both steady and oscillatory propagating fingers previously observed in experiments in axially uniform channels each containing a centred step-like occlusion. We find that a depth-averaged model can reproduce all the finger propagation modes observed experimentally. In addition, the model reveals new modes for symmetric finger propagation. The inclusion of a spatially variable channel depth in the depth-averaged equations leads to: (i) a variable mobility coefficient within the fluid domain due to variations in viscous resistance of the channel; and (ii) a variable transverse curvature term in the dynamic boundary condition that modifies the pressure jump over the air-liquid interface. We use our model to examine the roles of these two distinct effects and find that both contribute to the steady bifurcation structure, while the transverse curvature term is responsible for the distinctive oscillatory propagation modes.

Key words: bifurcation, bubble dynamics, Hele-Shaw flows

\section{Introduction}

The classical problem of Saffman \& Taylor (1958) concerns the propagation of a long air finger into a fluid-filled Hele-Shaw cell, i.e. a channel of constant rectangular cross-section whose width $W^{*}$ is much greater than its height $H^{*}$. Typically, if the fluid is removed at a constant flow rate, a single, steadily propagating air finger develops that is symmetric about the vertical midplane of the channel. By contrast with these unique symmetric families of fingers, recent experiments (de Lózar et al. 2009; Pailha et al. 2012; Hazel et al. 2013) have found complex dynamics in situations where rectangular channels with aspect ratios $\alpha=W^{*} / H^{*}$ in the range 4.5-12 are partially occluded by a centred rectangular block, which imposes a step-like reduction in the channel depth and has fractional width and height, $w=w^{*} / W^{*}$ and $h=h^{*} / H^{*}$, respectively. The experimental channel geometry is illustrated in figure 1 and observed finger shapes are shown in figure 2. In addition to the symmetric fingers as in figure $2(a, b)$, steadily propagating asymmetric fingers as in figure $2(c)$ were also found, together with distinctive oscillatory propagation modes, figure $2(d)$, in which

$\dagger$ Present address: Department of Mathematics, Imperial College London, London SW7 2AZ, UK. Email address for correspondence: alice.thompson1@imperial.ac.uk 

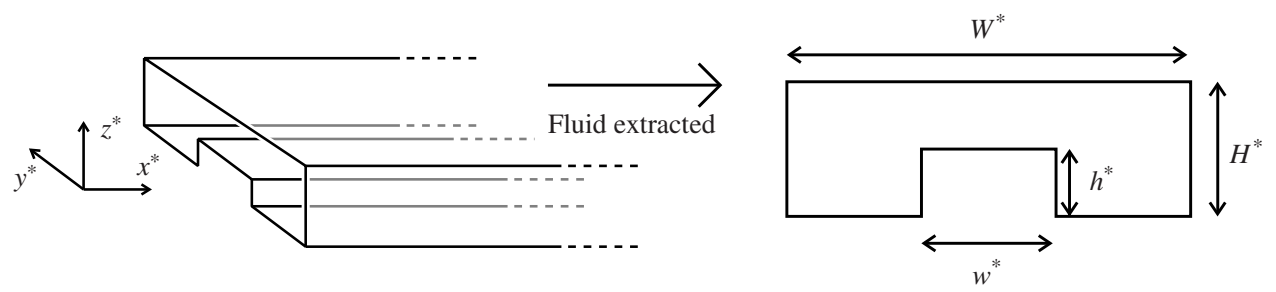

FIGURE 1. Schematic diagram of the channel geometry used in the experiments of de Lózar et al. (2009), Pailha et al. (2012) and Hazel et al. (2013). The channel cross-section, shown on the right, is independent of the axial coordinate $x^{*}$. The dimensional outer width and height of the cross-section are $W^{*}$ and $H^{*}$, respectively. The cross-section is occluded by an obstacle of dimensional width $w^{*}$ and height $h^{*}$ placed on one of the long sides of the cross-section. The channel is initially filled with viscous fluid, which is withdrawn at a constant volume flux from one end while the other end is left open to the air, resulting in the propagation of an air finger along the channel. In this paper, we examine the case where $\alpha \equiv W^{*} / H^{*} \gg 1$; we take a smoothed channel height profile, such as that shown in figure 4 , and depth-average in the $z^{*}$ direction.

(a)

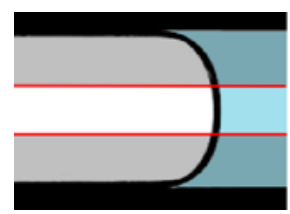

(b)

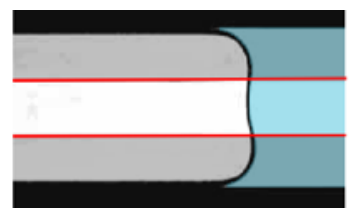

(c)

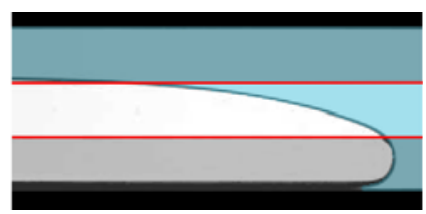

$(d)$

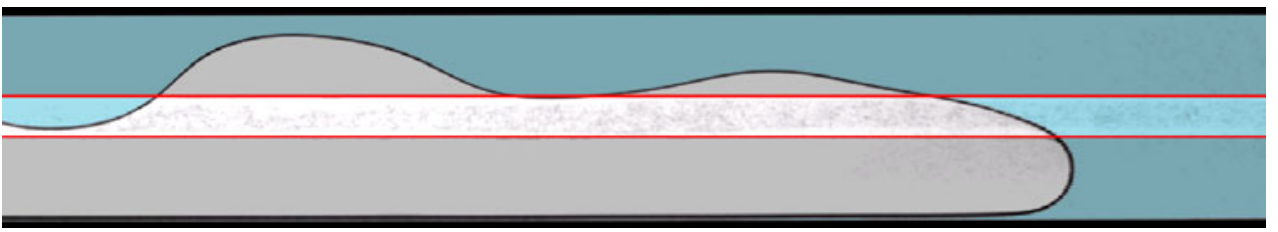

FIgURE 2. (Colour online) Top-view experimental images showing different modes of finger propagation at distinct parameter values: (a) steadily propagating symmetric 'round-nosed' finger; $(b)$ steadily propagating symmetric 'double-tipped' finger; $(c)$ steadily propagating asymmetric finger (de Lózar et al. 2009); and (d) an instantaneous snapshot of a time-dependent 'oscillatory' finger (Pailha et al. 2012), in which the finger tip sheds a spatially periodic disturbance as it propagates. The two edges of the obstacle are marked with horizontal lines, with lighter shading over the obstacle itself. The flow is driven by withdrawing fluid from the channel at a controlled volume flux ahead of the propagating finger tip. Throughout this paper, figures are shown with air fingers propagating from left to right.

the tip of the finger propagates with an almost-constant shape and speed but leaves behind a spatially varying periodic disturbance that remains essentially stationary in the laboratory frame.

An experimental bifurcation diagram of the finger propagation modes observed by Hazel et al. (2013) in different channels is shown in figure 3. A dimensionless finger tip speed $U$ is represented as a function of the dimensionless flow rate $Q$, which is the natural parameter for the depth-averaged system (see $\$ 2.1$ for definitions). Symmetry breaking is observed in the experiments, as first shown by de Lózar et al. (2009), who 


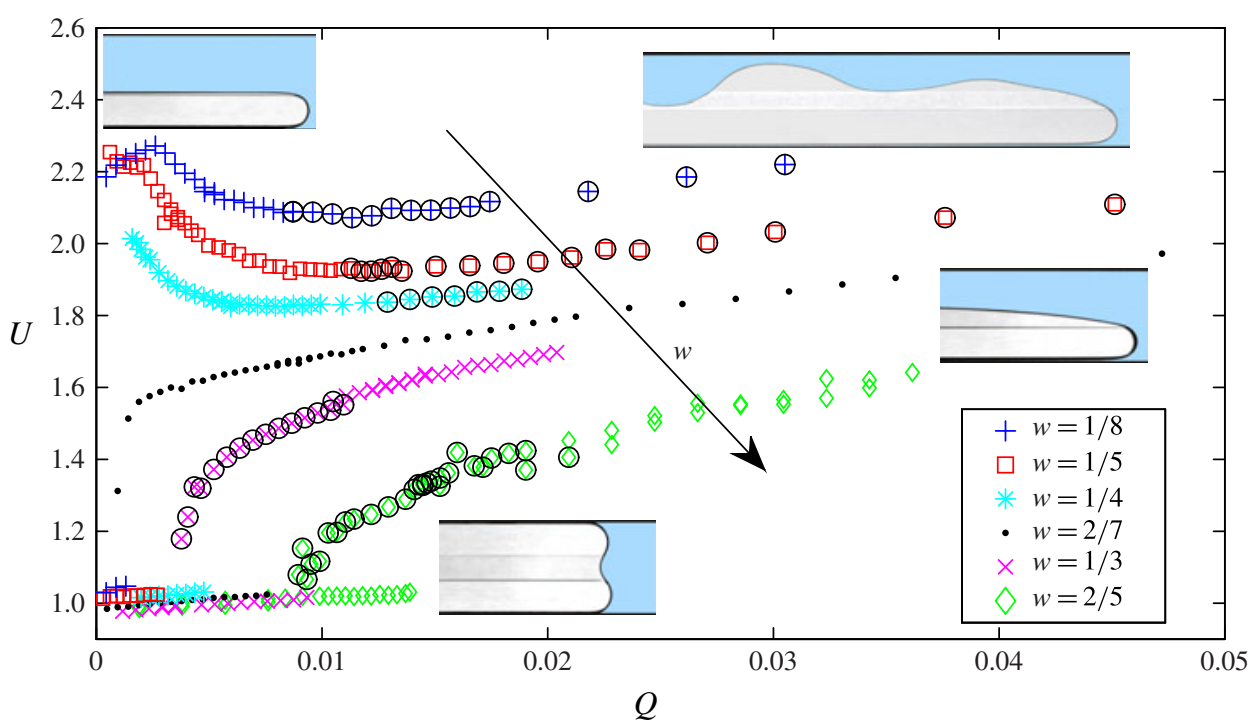

FIGURE 3. (Colour online) Experimental bifurcation diagram for a range of channels from data reported in figure 3 of Hazel et al. (2013), replotted in terms of the dimensionless parameter $Q=\mu U_{0}^{*} / \gamma$, a quantity proportional to the flow rate, and the resulting finger speed $U=U^{*} / U_{0}^{*}$, which are natural variables for the depth-averaged model described in $\S 2.1$. Here $\mu$ is the dynamic viscosity of the fluid, $\gamma$ is the surface tension at the air-liquid interface and $U_{0}^{*}$ is a representative speed of the liquid far ahead of the finger tip. Oscillatory propagation modes are indicated with a circle. In these experiments, three channel dimensions are fixed: $H^{*}=3.07 \pm 0.01 \mathrm{~mm}, h^{*}=1.50 \pm 0.02 \mathrm{~mm}$ and $w^{*}=4.50 \pm$ $0.01 \mathrm{~mm}$, but the channel width $W^{*}$ is varied, leading to the different values of $w \equiv w^{*} / W^{*}$ shown here.

uncovered a symmetry-breaking pitchfork bifurcation that evolves from supercritical to subcritical as the occluding block height increases. Symmetric propagation modes are observed only at very low flow rates, while for sufficiently high flow rates the propagation mode is always asymmetric and steady. These two regimes are bridged by a range of possible bifurcations that depend on channel geometry (Hazel et al. 2013).

For channels containing relatively wide, high obstacles $(w>1 / 4$ and $h=1 / 2$ in figure 3), a reduction in the flow rate from an initially asymmetric finger eventually leads to a symmetric solution. For channels with relatively narrow, high obstacles ( $w \leqslant 1 / 4$ and $h=1 / 2$ in figure 3), the fingers remain asymmetric as the flow rate is decreased, localising on one side of the obstacle at very low flow rates. This difference in observed behaviour with decreasing flow rate persists in the static limit $(Q=0)$, where both symmetric and localised asymmetric configurations can be found for sufficiently high and narrow obstacles; see Hazel et al. (2013).

Oscillatory propagation modes arise at intermediate flow rates for all but one of the channels represented in figure 3. Pailha et al. (2012) proposed a surface tension-driven mechanism to explain the oscillatory behaviour: if the edge of the finger passes over the occluding block, the finger can expand to fill the increased vertical space in the deeper part of the channel, resulting in a rapid decrease in local cross-sectional curvature; this decrease in curvature induces a local increase in fluid pressure, which rapidly drives the interface away from the occlusion towards the side-wall of the channel. Based on the measured logarithmic scaling of the period of the oscillations, 
Pailha et al. further conjectured that a global homoclinic connection gives rise to the oscillatory propagation modes.

The experimental results shown in figure 3 are for millimetric channels with Bond number $B o \equiv \rho g H^{* 2} / 4 \gamma=1.06$, where $\rho$ is the fluid density, $g$ is the acceleration due to gravity and $\gamma$ is the coefficient of surface tension. Thus gravitational effects should be comparable to surface tension. However, experiments for finger propagation in micro-scale channels where $B o=0.02$ also reveal similar multiple propagation modes (Jisiou et al. 2014), suggesting that gravity is not necessary to induce these bifurcations.

The aims of the current study are the following: firstly, to develop a model that qualitatively reproduces the diverse range of propagation modes observed in experiments; secondly, to use the model to explore the solution structure of the system; and, thirdly, to investigate the physical mechanisms that underlie the observed behaviour. In this paper, we study a depth-averaged model for finger propagation in the partially occluded channel. We base our model on the Saffman-Taylor model for finger propagation with non-zero surface tension described in McLean \& Saffman (1981). In rectangular channels, this depth-averaged model has been shown to be in quantitative agreement with numerical simulations of the three-dimensional free-surface Stokes equations for $\alpha \geqslant 8$ (de Lózar, Juel \& Hazel 2008). Moreover, the dynamics of this model are well understood for rectangular channels, and so it provides an excellent foundation for studying local variations in channel depth. There are two reasons for preferring such an analysis to direct simulation of the full three-dimensional system.

Firstly, our model allows us to explicitly decouple the roles of viscosity and surface tension in the partially occluded region. A local reduction in the channel height will increase both the viscous resistance of the channel and the transverse interfacial curvature. In the model, the first effect leads to a variable mobility coefficient in the governing pressure equation, whereas the second effect arises only in the dynamic boundary condition. We can therefore use the model to test which of these physical mechanisms is responsible for particular finger behaviours. We will show that both effects contribute to the bifurcation structure for steadily propagating fingers, while a spatially variable transverse curvature term in the dynamic boundary condition is the driving mechanism for the oscillatory propagation modes.

The second reason is simple numerical expediency: the depth-averaged system is two-dimensional, yielding a much smaller discretised system. In addition, depthaveraging avoids meshing the complicated three-dimensional fluid domain. In formulating this depth-averaged model, we make a number of assumptions regarding the behaviour of the thin films of fluid above and below the air finger, which build on previous models of Saffman-Taylor fingering (McLean \& Saffman 1981). Although three-dimensional simulations would be required to fully resolve the thin-film regions and enable quantitative comparison with experiments, we find that the two-dimensional model is remarkably effective in reproducing finger propagation qualitatively.

Depth-averaged models for finger propagation in wide channels were first suggested by Saffman \& Taylor (1958). In the zero-surface-tension limit, they found both symmetric and asymmetric families of fingers that satisfy the model equations. The degenerate selection problem is regularised by the inclusion of non-zero surface tension as a singular perturbation from a beyond-all-orders asymptotic analysis, which has been reviewed by Saffman (1986), Homsy (1987), McCloud \& Maher (1995) and Tanveer (2000), among others. The salient result for our purposes is that small but non-zero surface tension leads to the selection of a unique finger, which is symmetric 
and occupies half the width of the channel. For non-zero surface tension, McLean \& Saffman (1981) showed that the depth-averaged system can be characterised by a single dimensionless parameter $B=\gamma /\left(3 \mu U^{*} \alpha^{2}\right)$, where $\mu$ is the fluid viscosity and $U^{*}$ is the steady finger propagation speed. McLean \& Saffman solved the system numerically and found a single steady finger shape for each positive $B$. Other, wider, symmetric solutions to McLean \& Saffman's system were found by Romero (1982) and Vanden-Broeck (1983). For $B \ll 1$, all the fingers tend towards the symmetric half-width finger. As $B$ increases, the finger widens, eventually filling the whole channel as $B \rightarrow \infty$.

The steady finger shapes found by McLean \& Saffman are linearly stable for modest $B$ and are thought to be linearly stable whenever the surface tension is non-zero (Kessler \& Levine 1986; Tanveer 1987; Casademunt 2004). However, for noisy systems with small $B$, the interface is unstable to tip-splitting behaviour both experimentally (Park \& Homsy 1985; Maxworthy 1987; Tabeling, Zocchi \& Libchaber 1987) and numerically (Casademunt 2004). Tip splitting is followed by competition between fingers; once one finger begins to propagate ahead of the others, it screens the others and prevents them from growing. The dominant finger then grows until it becomes unstable to tip splitting, and the process repeats.

We note that the previously described partially occluded channel experiments were conducted at relatively large values of $B$, so that all putative bifurcations are distinct from the finger selection problem at zero surface tension, and the non-symmetric fingers (de Lózar et al. 2009) are unlikely to be directly related to low-surface-tension tip splitting.

A traditional application of Saffman-Taylor fingering is oil extraction from porous media, where the dynamics is governed by analogous equations. Interactions between viscous flow, surface tension and confined geometry are also very relevant when considering bubble and drop transport in microfluidic devices. Indeed, the use of constrictions in the channel height to obtain multiple finger propagation modes is related to the practical use of expansions in channel height to restrict the motion of finite-sized bubbles and drops, in the form of 'rails' and 'anchors' (Abbyad et al. 2011; Dangla, Lee \& Baroud 2011; Nagel, Brun \& Gallaire 2014). Localised channel-height expansions in microfluidic devices allow the bubble to expand vertically, thus reducing its surface energy and stabilising the bubbles against an imposed flow (Dawson, Lee \& Juel 2013).

Our main focus in this paper is to explore the bifurcation structure of a viscous two-phase displacement flow in a Hele-Shaw channel with centred, axially uniform depth variations. We derive the depth-averaged model in $\S 2.1$, with an emphasis on how it is altered by the variable channel height profile. The numerical implementation of the model using the open-source finite element library oomph-lib (Heil \& Hazel 2006 ) is discussed in $\S 2.2$. In $\S 3$, we describe steady propagation modes and their bifurcations. Oscillatory propagation modes and their relationship to the bifurcation structure for steady solutions are discussed in $\S 4$. In $\S 5$ we discuss the effect of obstacle smoothing on the linear stability of oscillatory disturbances to a straight interface aligned parallel to the channel side-wall, which is a simple model for the side of the finger. Finally, in $\S 6$, we conclude with a summary of our results and the shortcomings of the model, along with a discussion of other studies of 'perturbations' to the Saffman-Taylor fingering problem. 


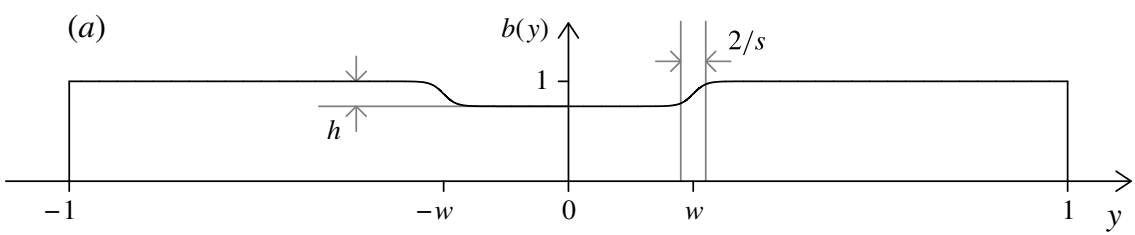

(b)

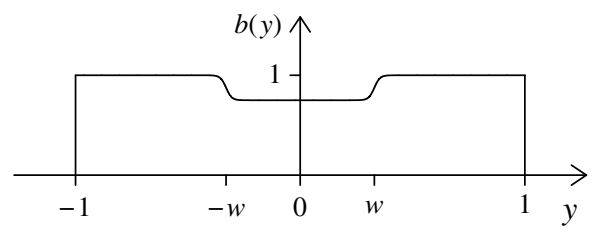

FIGURE 4. Plot of the smoothed channel cross-section from (2.1) for: (a) a main channel with $\alpha=10, w=0.25, s=40$ and variable $h$, shown here with $h=0.25$; and $(b)$ an alternative channel with $\alpha=4.5, w=0.33$ and $s=40$, used as a comparison case for stability calculations as discussed in $\S 4.2$, also shown here with $h=0.25$. Both crosssections are drawn to scale. The smoothing of the step is controlled by the sharpness parameter $s$.

\section{Problem formulation}

\subsection{Derivation of depth-averaged equations}

We develop a two-dimensional model for finger propagation in a wide channel with a spatially varying height profile. Using Cartesian coordinates, the coordinate $x^{*}$ is aligned with the channel axis, and $y^{*}$ and $z^{*}$ span the width and height of the cross-section, respectively; see figure 1 . The notional flow domain is $-\infty<x^{*}<\infty$, $-W^{*} / 2<y^{*}<W^{*} / 2$ and $0<z^{*}<b^{*}\left(y^{*}\right)$, where an asterisk is used to indicate dimensional quantities; note that the range of $x^{*}$ will be truncated in our numerical simulations. The channel has outer width $W^{*}$ and maximum height $H^{*}$, with aspect ratio $\alpha \equiv W^{*} / H^{*} \gg 1$; we non-dimensionalise $x^{*}$ and $y^{*}$ on the scale $W^{*} / 2$ and $b^{*}$ on the scale $H^{*}$. We choose a non-dimensional depth profile $b=b(y)$ with a smoothed step-like occlusion as shown in figure 4 , given by

$$
b(y)=1-\frac{h}{2}[\tanh s(y+w)-\tanh s(y-w)],
$$

where $h$ is the height of the step, $w$ is the width of the step and $s$ is a parameter governing the smoothing of the step. If $h=1$, the step occupies the full channel height; if $h=0$, there is no step and the channel has constant depth; and if $h<0$, the step is an expansion of the channel height such as for the grooved system studied experimentally by Rabaud, Couder \& Gerard (1988). We focus in this paper on the bifurcations that occur as the step height $h$ increases from zero.

We assume that the flow is driven by a uniform pressure gradient $-G^{*} \boldsymbol{e}_{x}$ far ahead of the finger tip; for the depth-averaged system, the imposed pressure gradient is equivalent to a time-independent volume flux, analogous to the withdrawal of fluid at constant flow rate in the experimental system. We non-dimensionalise the pressure $p^{*}$ on the scale $G^{*} W^{*} / 2$ and the two components of the depth-averaged horizontal velocity $\boldsymbol{u}^{*}$ on the scale $U_{0}^{*} \equiv G^{*} H^{* 2} / 12 \mu$, where $\mu$ is the dynamic viscosity of the fluid. Finally, we choose the natural time scale so that $t^{*}=W^{*} / 2 U_{0}^{*} t$. We apply the 
lubrication approximation (Reynolds 1886) to obtain the following equations at leading order in $\alpha^{-1}$ for the dimensionless depth-averaged fluid velocity:

$$
\boldsymbol{u}=-b^{2} \nabla p \quad \text { and } \quad \boldsymbol{\nabla} \cdot(b \boldsymbol{u})=0,
$$

which combine to give

$$
\nabla \cdot\left(b^{3} \nabla p\right)=0,
$$

where $\nabla=(\partial / \partial x, \partial / \partial y)$ is the horizontal gradient operator.

If $b(y)$ is constant, then (2.3) reduces to $\nabla^{2} p=0$, which allows the use of conformal mapping techniques, as exploited by Saffman \& Taylor (1958) and McLean \& Saffman (1981) for channels of constant depth. However, for channels with an arbitrary height profile $b(y)$, these analytical techniques no longer apply. Instead, we use a finite element method, which allows us to solve the discrete version of (2.3) directly for any height profile $b(y)$.

On the channel boundaries, we enforce the no-permeability condition $\boldsymbol{n} \cdot \boldsymbol{u}=0$, where $\boldsymbol{n}$ is a two-dimensional vector normal to the appropriate boundary. We note that this leaves the tangential component of $\boldsymbol{u}$ free to vary, resulting in non-zero slip along the side-walls of the channel, even far ahead of the finger; the no-slip boundary condition of the full Stokes equations does not occur at leading order in $\alpha^{-1}$.

In formulating the kinematic boundary condition on the finger interface for the depth-averaged system, we make two assumptions about the interface shape in the transverse plane, which contains $\boldsymbol{e}_{z}$ and is normal to the advancing interface: firstly that the films deposited above and below the finger have zero thickness, and secondly that the finger shape in the transverse plane does not change as the finger advances. The kinematic boundary condition for the depth-averaged system is then

$$
\frac{\partial \boldsymbol{R}}{\partial t} \cdot \boldsymbol{n}=\boldsymbol{u} \cdot \boldsymbol{n},
$$

where $\boldsymbol{R}=(x, y)$ is the position of the interface, so that the interface moves with the average normal velocity of the fluid. We note that (2.4) is a depth-averaged expression and does not specify the three-dimensional velocity at the interface itself. Some threedimensional fluid rearrangement is required, typically over a distance comparable to the channel height, in order to reconcile a steadily propagating interface with the parabolic flow profile far ahead of the interface; this secondary flow, like the no-slip boundary condition, is not evident at leading order in $\alpha^{-1}$.

For the dynamic boundary condition, we assume that within the transverse plane, the air-fluid interface takes the shape of a semicircle with radius $b(y) / 2$. The finger then completely fills the channel height, and the fluid perfectly wets the upper and lower walls of the channel when they are horizontal. We neglect the corrections to the semicircular shape proposed by Park \& Homsy (1984) and Reinelt (1987) for the case of very strong surface tension. We will further assume that the pressure jump across the finger interface is due to surface tension only and so

$$
p_{b}-p=\frac{1}{6 \alpha Q}\left(\frac{2}{b(y)}+\frac{2 \kappa}{\alpha}\right),
$$

where $p_{b}$ is the constant pressure inside the air finger, $Q \equiv \mu U_{0}^{*} / \gamma$ is a nondimensional flow rate, $\gamma$ is the coefficient of surface tension and $\kappa$ is the nondimensional curvature of the interface in the $(x, y)$ plane. There are two components 


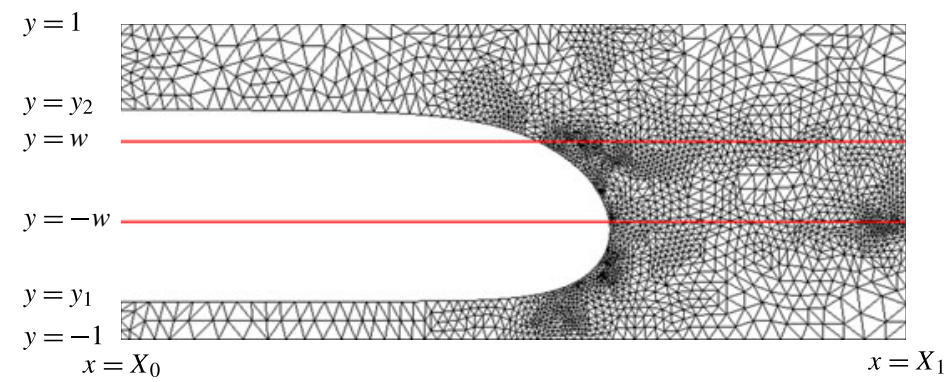

FIgURE 5. (Colour online) A typical computational domain discretised with an unstructured triangular mesh. The channel is truncated ahead of and behind the tip of the finger, at $x=X_{0}$ and $x=X_{1}$, respectively. The finger is characterised by its dimensionless speed $U$ and offset $\delta \equiv\left(y_{1}+y_{2}\right) / 4$, which are both dependent on the coordinates $y_{1}$ and $y_{2}$ at which the air-fluid interface meets the truncation point $x=X_{0}$.

to the interface curvature: the lateral curvature $\kappa$ and the transverse curvature $2 / b(y)$, which is the curvature of the semicircular interface constrained within the channel height $b(y)$. We note that our use of the boundary condition (2.5), proposed by McLean \& Saffman (1981), is not rational in a strict asymptotic sense owing to the presence of terms involving $\alpha^{-1}$ and $\alpha^{-2}$. Nonetheless, the boundary condition (2.5) is a simple way of incorporating surface tension, and is known to lead to quantitative agreement with three-dimensional simulations in rectangular tubes when $\alpha \geqslant 8$ (de Lózar et al. 2008). For these rectangular tubes, the lateral curvature term in (2.5) is needed to avoid the degenerate selection problem which arises for zero surface tension, whereas the transverse curvature term is constant and does not affect the interface shape. However, when $b$ is not constant, the transverse curvature $2 / b(y)$ varies across the channel and we find that it can have a dramatic effect on the interface shape.

We aim to characterise steadily propagating modes as well as propagating oscillatory states. We therefore work in a frame moving with the tip of the finger, by subtracting a spatially constant velocity $\boldsymbol{U}_{F}=(U(t), 0)$ from the interface motion, so that $\boldsymbol{u}=$ $-\boldsymbol{U}_{F}-b^{2} \nabla p$, with $U(t)$ determined by the condition that the $x$-coordinate of the finger tip remain fixed in the moving frame.

Far behind the finger tip, for steady solutions we enforce $\partial p / \partial x \rightarrow 0$. For oscillatory states, we find that retaining the boundary condition $\partial p / \partial x=0$ leads to convergence problems when the interface crosses the smoothed edge of the occlusion at the very end of the domain. Instead, for time-dependent calculations, we allow $\partial p / \partial x$ to be non-zero on each outflow, with $\partial p / \partial x=G_{1}(t)$ and $G_{2}(t)$ on the two boundaries $-1<y<y_{1}$ and $y_{2}<y<1$, respectively, as shown in figure 5. The pressure gradient corrections $G_{1,2}$ are determined numerically as part of the solution, in order that the interface position $y(x, t)$ satisfies the boundary condition

$$
\frac{\partial y}{\partial t}=U \frac{\partial y}{\partial x}
$$

at the two points $y=y_{1,2}(t)$ where the interface leaves the domain.

Thus, the complete system of equations and boundary conditions required to determine the pressure in the fluid domain $\Omega$, with $\partial \Omega_{b}$ denoting the finger boundary, is

$$
\nabla \cdot\left(b^{3} \nabla p\right)=0 \quad \text { in } \Omega,
$$




$$
\begin{aligned}
& \boldsymbol{n} \cdot \boldsymbol{R}_{t}+\boldsymbol{n} \cdot \boldsymbol{U}_{F}+b^{2} \boldsymbol{n} \cdot \nabla p=0 \quad \text { on } \partial \Omega_{b}, \\
& p-p_{b}+\frac{1}{3 \alpha Q}\left(\frac{1}{b}+\frac{\kappa}{\alpha}\right)=0 \quad \text { on } \partial \Omega_{b} \text {, } \\
& \frac{\partial p}{\partial y}=0 \quad \text { on } y= \pm 1, \\
& \frac{\partial p}{\partial x}=G_{1,2} \quad \text { on } x=X_{0}, \\
& \frac{\partial p}{\partial x}=-1 \quad \text { on } x=X_{1} \text {. }
\end{aligned}
$$

Here $X_{0}$ and $X_{1}$ are the truncation coordinates behind and ahead of the finger tip, respectively. For steady calculations, we typically take $X_{0}=-6$ and $X_{1}=4$, with the tip located at $x=0$. For oscillatory calculations, we typically allow double the usual truncation distance behind the finger tip. We set $G_{1,2}=0$ for steady calculations.

The parameters in the system are $\alpha$ and $Q$, which appear in the dynamic boundary condition, and $h, w$ and $s$, which are used to define the depth profile $b(y)$. In this paper, we primarily consider bifurcations as $Q$ and $h$ vary for a channel with $\alpha=10$ and $w=0.25$; we take the smoothing parameter $s$ to be 40 throughout, leading to the smoothed profiles shown in figure 4 .

\subsection{Implementation in oomph-lib}

We formulate the system (2.7)-(2.12) as a weak problem and solve the equations numerically using a finite element discretisation implemented in the 'object-oriented multi-physics finite element library' oomph-lib (Heil \& Hazel 2006). We calculate both steadily propagating and time-dependent modes, and use a combination of arclength continuation, bifurcation tracking and linear stability analysis to explore the bifurcation structure for steady propagation modes.

After multiplying by a test function $\psi$ and integrating by parts, the weak form of the bulk pressure equation (2.7) becomes

$$
\int_{\Omega} b^{3}(y) \nabla p \cdot \nabla \psi \mathrm{d} \Omega=\int_{\partial \Omega} \psi b^{3}(y) n \cdot \nabla p \mathrm{~d} s .
$$

We use an isoparametric Galerkin method, so that the functions which interpolate the pressure and unknown nodal positions are the same as the test functions, in this case piecewise-quadratic shape functions. The equations (2.8)-(2.12) give conditions for $\boldsymbol{n}$ • $\nabla p$ on each boundary of the fluid domain and are used on the right-hand side of (2.13).

The deformation of the interface can be large (figure 10 shows an extreme example), so for direct calculations of steady and time-dependent behaviour we use an unstructured 'pseudo-elastic' mesh: the deformation of bulk nodes in response to interface motion is determined by treating the interior nodes themselves as material points in a fictitious elastic solid body (Sackinger, Schunk \& Rao 1996). The interior of the fluid domain is decomposed into triangular elements (see figure 5), with six nodes per element required for the quadratic interpolation of the fluid pressure. The interface deformation for the pseudo-elastic mesh is achieved by applying a normal stress to the solid equations at the free surface. This normal stress acts as a Lagrange multiplier, and is determined by the weak form of the dynamic boundary condition (2.9). In the bulk mesh, both the fluid pressure and the position coordinates are 
interpolated using piecewise-quadratic shape functions. However, the curvature term in (2.9) requires the second derivative of the interface position, which is not smooth for a piecewise-quadratic representation of the interface. We therefore project a weak derivative of the tangent vector onto a piecewise-quadratic space in order to obtain a continuous curvature term.

For direct time-dependent calculations, the time integration is implemented using the second-order backward differentiation formula BDF2. As the time derivatives in the problem occur only in the interface terms, we do not require any arbitrary LagrangianEulerian terms to account for the moving mesh.

After a number of continuation steps for steady solutions, or time steps for time-dependent calculations, individual elements within the bulk mesh can become highly deformed, leading to a poor-quality triangulation of the domain. In order to prevent mesh deterioration affecting the interface motion, we perform a complete mesh regeneration every few steps, generating a new triangulation based on the previous discretisation of the boundary when required by an estimate of the error within the fluid domain, the deformation of the elements themselves or a poor discretisation of the interface. We use a 'Z2' error estimator (Zienkiewicz \& Zhu 1992) based on the continuity of $\boldsymbol{u}=-b^{2} \nabla p$ between elements; this error estimator automatically results in finer elements along the edge of the step, as shown in figure 5. We choose target areas for the domain triangulation in response to the estimate of error within the fluid, and refine the interface in regions of high curvature. The fluid domain was typically discretised with around 2000 triangular elements; we confirmed the robustness of the results by varying the mesh resolution.

In order to compute the location of bifurcations, we formulate an extended system to track a single eigenmode with the required properties (Cliffe, Spence \& Tavener 2000). Both bifurcation tracking and linear stability calculations are relatively costly computations; for these calculations we usually replace the pseudo-elastic mesh with a more efficient 'spine' mesh using quadrilateral elements (de Lózar et al. 2008), in which the interface and bulk nodes are placed at specified fractions along vector spines. The spine mesh uses significantly fewer degrees of freedom than the pseudoelastic mesh, because at nodes in the domain interior we need only determine the fluid pressure. The spine mesh efficiently describes cases where the tip of the finger is not significantly deformed, e.g. for the shapes shown as insets in figure 7, but is not suitable for calculations with larger deformations because the spine directions must be chosen a priori and cannot easily be refined in specific regions.

For the linear stability analysis, we wish to determine the evolution of small perturbations to the interface of a steadily propagating finger. Our choice of non-dimensionalisation is important for the linear stability analysis: we keep the dimensionless flow rate $Q$ fixed, but allow perturbations to the speed $U$ of the finger tip, while still requiring the frame to exactly track the finger tip. Rather than first linearising and then discretising the equations, we use a numerical approach in which we analyse linear perturbations to the discretised equations. We begin this analysis by writing the equations for the spatially discretised system in the general form $\boldsymbol{R}(\dot{\boldsymbol{u}}, \boldsymbol{u})=0$, where the vector $\boldsymbol{u}$ contains all of the unknown variables in the discretised problem and $\dot{\boldsymbol{u}}=\partial \boldsymbol{u} / \partial t$. We use the same finite element discretisation for the spatial problem as for the calculations of steadily propagating fingers and time-dependent propagation. As the time-dependent system (2.7)-(2.12) is linear with respect to the time-derivative terms, we may write $\boldsymbol{R}$ without loss of generality as

$$
\boldsymbol{R}=\boldsymbol{M}(\boldsymbol{u}) \dot{\boldsymbol{u}}+\boldsymbol{F}(\boldsymbol{u})
$$

where $\boldsymbol{M}$ is the mass matrix. 


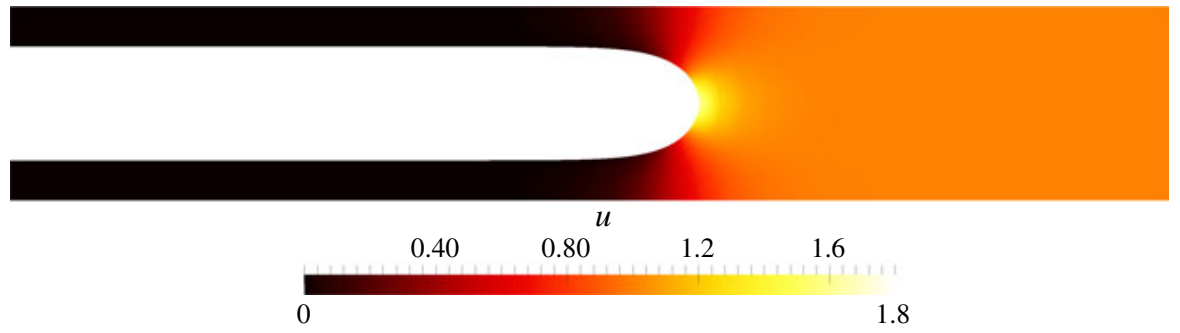

FIGURE 6. (Colour online) The unique steady finger shape for the unoccluded channel with $\alpha=10$ and $Q=0.08$. Here $U=1.70$, which corresponds to $\lambda=0.589$ and $\kappa=1.426$ in the variables of McLean \& Saffman (1981) and is consistent with their predictions for finger width to within $0.1 \%$. Throughout this paper, plots of this style are shaded by the axial velocity component $u$ and use the scale shown here unless otherwise specified. Note the finite slip along the channel side-walls at $y= \pm 1$. Far behind the tip of the finger, the interface is parallel to the channel walls, and the fluid is at rest.

The base state for our linear stability analysis is a steadily propagating finger represented by $\boldsymbol{u}_{0}$, where $\boldsymbol{F}\left(\boldsymbol{u}_{0}\right)=0$. We now examine the time evolution of a small perturbation to this steadily propagating finger, setting $\boldsymbol{u}=\boldsymbol{u}_{0}+\epsilon \boldsymbol{v}$. For $\epsilon \ll 1$, the requirement that $\boldsymbol{R}=0$ is equivalent to

$$
\boldsymbol{M}\left(\boldsymbol{u}_{0}\right) \dot{\boldsymbol{v}}+\boldsymbol{J}\left(\boldsymbol{u}_{0}\right) \boldsymbol{v}=0, \quad \boldsymbol{J}=\frac{\partial \boldsymbol{F}}{\partial \boldsymbol{u}},
$$

where $\boldsymbol{J}$ is the same Jacobian matrix as arises when solving for steadily propagating solutions. We decompose $\boldsymbol{v}$ as a sum of eigenvectors $\boldsymbol{v}_{n}$, each of which satisfies $\dot{\boldsymbol{v}}_{n}=$ $\lambda_{n} \boldsymbol{v}_{n}$; thus the eigenvectors are solutions to the generalised eigenvalue problem

$$
(\lambda \boldsymbol{M}+\boldsymbol{J}) \boldsymbol{v}=0 .
$$

The solution $\boldsymbol{u}_{0}$ for steady finger propagation is stable when the real part of $\lambda_{n}$ is negative for all eigenvalues.

The only time derivatives in the problem are the terms involving interface motion in the kinematic boundary condition (2.8), which is associated with the bulk pressure test function $\psi$ through (2.13). Hence, the mass matrix $\boldsymbol{M}$ is non-symmetric and significantly rank-deficient, which requires special attention as discussed in appendix A.

The code was first validated by comparison to the known McLean \& Saffman (1981) solutions for rectangular channels (see figure 6). Secondly, excellent agreement was achieved between results obtained with the two different types of mesh, which use completely different code to obtain discretised equations. Finally, selected calculations were performed at increased mesh resolution, and also by doubling the standard domain truncation distance. The standard domain was sufficient to resolve all the steadily propagating finger shapes exhibited in this paper. However, some truncation effects were detected for oscillatory propagation modes, which we discuss in $\S 4$.

The smoothed profile introduces a new parameter that does not arise in experiments, namely the sharpness parameter $s$ in (2.1) that controls the degree of smoothing, as indicated in figure 4 . We found that the bifurcation structure for steady solutions 
is remarkably robust with respect to variations in $s$. In particular, we tracked two bifurcations that occur at $h=0.05$ and $\alpha=10$ as $s$ was varied over the range 10-160. For each of these bifurcations, the critical value of $Q$ at the bifurcation point changed by less than $0.5 \%$ as $s$ was increased from 40 to 160 . Increasingly fine meshes are required to resolve solutions for large $s$, and thus calculations for very large $s$ are infeasible. Moreover, the assumptions underlying the model will break down once $s$ is sufficiently large. However, over the range of $s$ studied, our results indicate that the bifurcation points converge to constant critical values of $Q$, which are likely to remain unchanged as $s \rightarrow \infty$. As $s$ decreases, the critical values of $Q$ increase and diverge from the values obtained at $s=40$, displacing the bifurcation point in the same direction as when the obstacle is lowered. The value $s=40$ represents a compromise between the desire to obtain solution behaviour relevant to experiments where the step is very sharp, the limitations of our depth-averaged model and the resolution of the numerical solutions. For the oscillatory propagation modes, the sharpness parameter $s$ influences the wavelength of oscillations, as discussed in $\S 4$.

\section{Solutions for steadily propagating fingers}

If $h=0$ and $b=1$, our model reduces to that of McLean \& Saffman (1981) for finger propagation in rectangular channels with non-zero surface tension, and we have verified that our numerical scheme correctly predicts the finger width, with a typical finger shape as shown in figure 6 . We use the $h=0$ solutions to obtain steady solutions for larger obstacle heights by continuation in $h$.

We find that our depth-averaged system exhibits finger propagation modes and an overall bifurcation structure that are remarkably similar to the behaviour observed experimentally (de Lózar et al. 2009; Pailha et al. 2012; Hazel et al. 2013). In this section we describe the steady solution structure as the dimensionless flow rate $Q$ and the obstacle height $h$ vary.

\subsection{Symmetry breaking}

For the very lowest flow rates at low obstacle heights, the steadily propagating finger shape closely resembles the finger shape for $h=0$. The finger is symmetric and passes over the obstacle into the unoccluded parts of the channel, there are no points of inflexion on the interface, and the finger is linearly stable. As $Q$ is slowly increased, we find that the finger narrows slightly but that the shape still closely resembles the finger shape for the unoccluded channel. At $Q=Q_{P}$, two asymmetric finger shapes emerge via a symmetry-breaking pitchfork bifurcation.

The flow is driven by a fixed volume flux, which must be equal to the rate of increase of the air volume, and fluid motion is concentrated immediately ahead of the advancing finger tip. An asymmetric finger drives less fluid through the region of reduced mobility (the narrow gap), and the dissipation is lower than for the corresponding symmetric state. Thus, an asymmetric finger will be preferred for sufficiently high flow rates, when the relative difference in viscous dissipation between the symmetric and asymmetric states is greatest. In the model, the local variation in mobility affects the finger motion through the kinematic boundary condition (2.8), which shows that, for a fixed pressure gradient, increasing $b$ leads to an increase in $\boldsymbol{U}_{F}$, hence providing a mechanism for symmetry breaking. An alternative mechanism arises in combination with the dynamic boundary condition (2.9), because the reduction in transverse curvature as $b$ increases leads to a local increase in fluid pressure and hence an increase in axial pressure gradient and therefore $\boldsymbol{U}_{F}$, again 
(a)

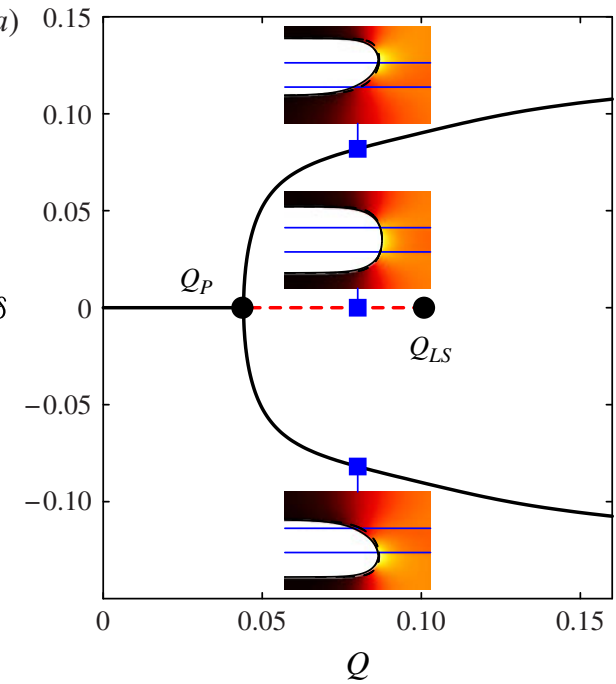

(b)

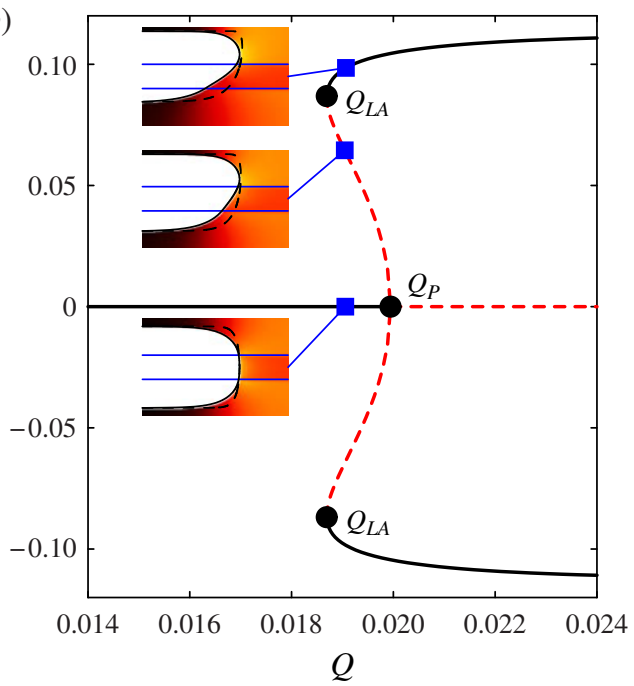

FIGURE 7. (Colour online) The finger offset measure $\delta \equiv\left(y_{1}+y_{2}\right) / 4$ (see figure 5) as a function of $Q$, which shows a perfect symmetry-breaking pitchfork bifurcation at $Q=Q_{P}$ for $(a) h=0.05$, where the pitchfork bifurcation is supercritical, and $(b) h=0.11$, where the pitchfork bifurcation is subcritical. For $h=0.11$, the two pairs of asymmetric branches merge at a pair of limit points at $Q=Q_{L A}$. The diagram for $h=0.05$ also indicates a limit point at $Q=Q_{L S}$ between two different symmetric solution branches, which we shall discuss in §3.2. Taylor \& Saffman (1959) derived an analytical expression for a two-parameter family of steadily propagating fingers at zero surface tension. The dashed lines in the inset fingers show Taylor-Saffman shapes with the two parameters chosen to match the finger offset and width.

through the kinematic condition. We have confirmed that each mechanism in isolation can lead to symmetry breaking (see $\S 3.2$ ) but that the viscous mechanism is required to stabilise asymmetric solutions at large flow rates.

We quantify the degree of asymmetry by using the offset from the channel centre, $\delta \equiv\left(y_{1}+y_{2}\right) / 4$, where $y_{1}$ and $y_{2}$ are the $y$-coordinates of the two points at which the air-liquid interface meets the domain boundary, illustrated in figure 5. This measure of asymmetry is analogous to the value of the offset $\delta$ reported in the experimental literature for this problem (de Lózar et al. 2009; Pailha et al. 2012; Hazel et al. 2013). Figure 7(a) shows the bifurcation diagram for $h=0.05$ obtained by plotting $\delta$ as a function of $Q$. The supercritical pitchfork bifurcation occurs at $Q=Q_{P}=0.044$, where the symmetric solution branch exchanges stability with the two emerging asymmetric solution branches. The tip shapes for symmetric and asymmetric fingers beyond the pitchfork at $Q=0.08$ are shown as insets in figure $7(a)$.

We explicitly track the location of the pitchfork bifurcation $Q_{P}$ as a function of obstacle height $h$, and the results are shown by the solid line in figure 8 . We find that the pitchfork bifurcation migrates towards smaller $Q$ as $h$ is increased and appears to persist as $h \rightarrow 0$, where $Q_{P} \rightarrow \infty$ in this limit. In fact, this is precisely the low-surface-tension limit in which the system is degenerate, and it is possible that the overall bifurcation structure arises through regularisation of the degeneracy by the introduction of a well-defined spatial structure. Taylor \& Saffman (1959) showed that at zero surface tension, the depth-averaged model has a two-parameter 


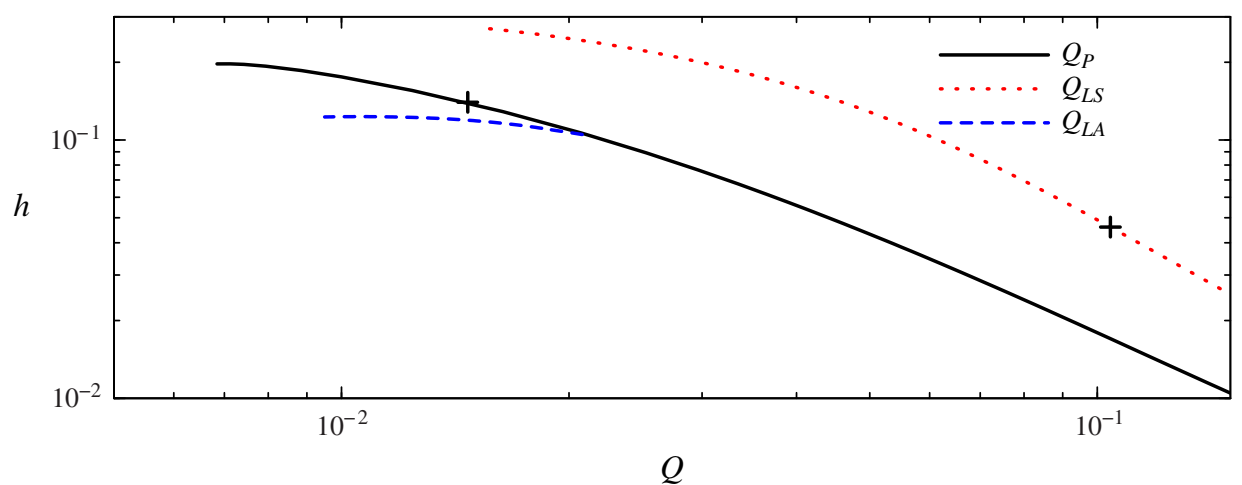

FIgURE 8. (Colour online) The location of the pitchfork bifurcation $Q_{P}$, the asymmetric limit point $Q_{L A}$ for heights where the pitchfork bifurcation is subcritical, and the symmetric limit point $Q_{L S}$, as $h$ varies with fixed $\alpha=10$ and $w=0.25$. The crosses mark the points at which the finger shapes at the bifurcations transition from 'round-nosed' to 'double-tipped' as $h$ is increased; see $\S 3.2$. The asymmetric limit point $Q_{L A}$ appears to show a turning point in $h$, equivalent to a transcritical bifurcation, close to the lowest $Q$ for which this bifurcation is shown. The lowest values of $Q$ correspond to the limit of resolution of thin films which develop along one or both sides of the finger. The implications of a transcritical bifurcation and the thin-film effects are discussed in $\S 3.4$.

family of solutions, with arbitrary offset and width. We compare our tip shapes in the partially occluded channel to the finger shapes for these zero-surface-tension solutions at matched finger offset and width, as shown by the dashed lines in the insets of figure 7. The finger shapes for the zero-surface-tension solutions are similar to our solutions for $h=0.05$ and $Q=0.08$, but the tip shapes deviate as $h$ increases, and are markedly different for $h=0.11$.

As $h$ increases, the symmetry-breaking pitchfork bifurcation transitions from supercritical to subcritical at $h \approx 0.103$. At heights just above this transition, as illustrated for $h=0.11$ in figure 7(b), there is a finite range of $Q$ over which we can calculate two different branches of asymmetric fingers biased to one side of the channel, along with another pair from the reflection in $y=0$, and also the symmetric solution branch. Each pair of asymmetric branches is connected by a limit point at $Q=Q_{L A}<Q_{P}$, as indicated in figure 7(b). The asymmetric branches with the largest offset magnitude $|\delta|$ are stable for $Q>Q_{L A}$, while the symmetric branch is stable for $Q<Q_{P}$. These branches are connected for $Q_{L A}<Q<Q_{P}$ by unstable intermediate asymmetric branches. We track the asymmetric limit point $Q_{L A}$ for heights where the pitchfork bifurcation is subcritical; its location as $h$ varies is indicated by the dashed line in figure 8. The width of the hysteresis region $\left(Q_{L A}, Q_{P}\right)$ increases with $h$, so that the effects of hysteresis become more significant for higher obstacles. However, we can also observe a turning point in $h\left(Q_{L A}\right)$ at the lowest $Q$ shown in figure 8 , which is associated with a transcritical bifurcation, discussed in $\S 3.4$.

The symmetry-breaking behaviour described in this section is in excellent qualitative agreement with the experimental investigations of de Lózar et al. (2009). Firstly, our calculated tip shapes for asymmetric propagation modes are very similar to the experimental observations presented by de Lózar et al., an example of which is shown in figure 2(c). We also compare the nature of the symmetry-breaking bifurcation as $Q$ varies. We note that the experimental results are reported in terms of the capillary 


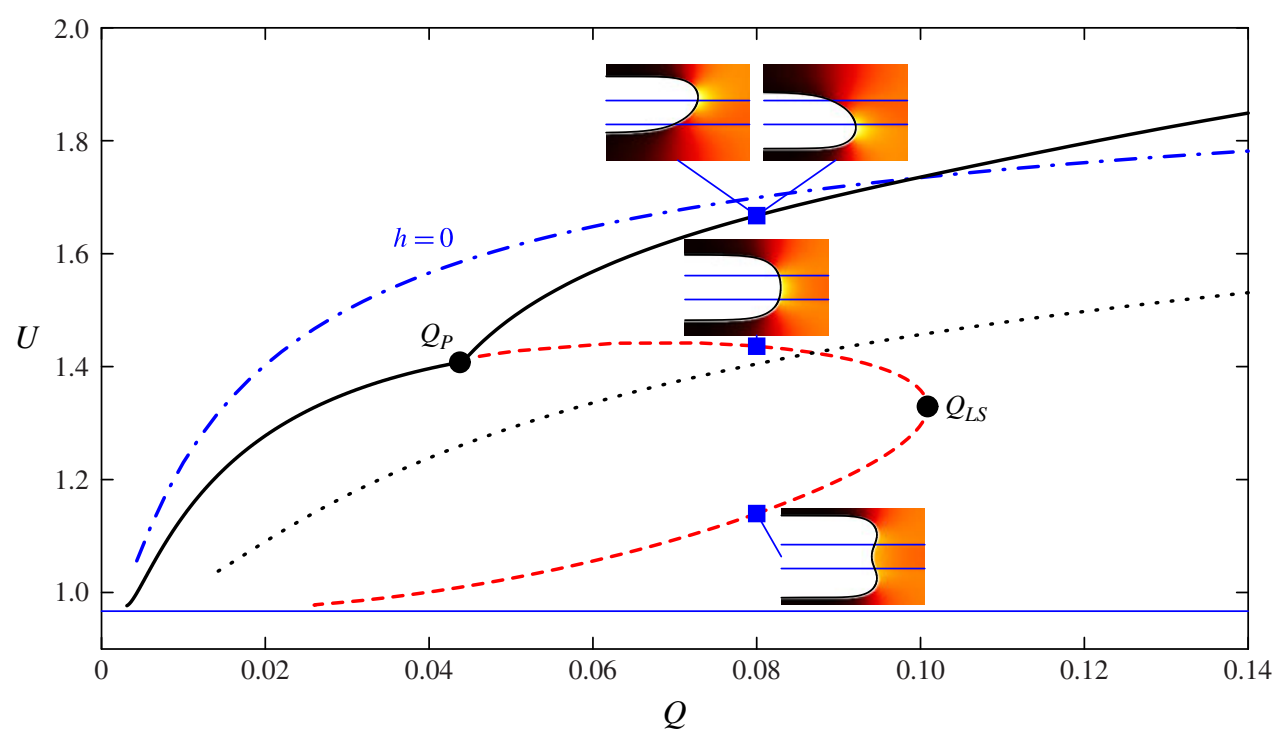

FIgURE 9. (Colour online) Finger tip speed $U$ as a function of $Q$ for $h=0.05$; this is an alternative projection of the bifurcation structure shown in figure $7(a)$. In particular, this projection allows us to observe the limit point at $Q=Q_{L S}$ between two different symmetric branches. The horizontal line at $U=0.967$ shows the propagation speed for fingers that fill the whole width of the channel. The behaviour of symmetric fingers for $Q>Q_{L S}$ is discussed in $\$ 3.3$, and leads us to calculate steady solutions for the propagation of doublefinger modes, as illustrated in figure $10(c)$, with speed shown for comparison here by the dotted line. We also show $U(Q)$ for an unoccluded channel with the same aspect ratio: the dash-dotted line indicates the solution family found by McLean \& Saffman (1981).

number based on the speed of the finger tip, $C a \equiv U Q$, rather than the controlled flow rate $Q$; but fortunately within the parameter range shown in figure 7 , we find that $1<U<2$ and increased $C a$ corresponds to increased $Q$. For both experiments and our model, the fingers with the largest offset magnitude occur at large $Q$, and the symmetry-breaking pitchfork becomes increasingly subcritical (the distance between $Q_{P}$ and $Q_{L A}$ increases) as $h$ increases.

\subsection{Multiple symmetric propagation modes}

The solid lines in the bifurcation diagram for $h=0.05$ shown in figure $7(a)$ present a consistent description of stable propagation modes, with stable symmetric fingers for $Q<Q_{P}$ and stable asymmetric fingers for $Q>Q_{P}$. However, in this diagram, the unstable symmetric branch apparently terminates beyond the pitchfork at the point marked $Q_{L S}$; this is a limit point between two branches of symmetric fingers that are indistinguishable using the measure $\delta$, which is zero for all symmetric fingers. We instead use the finger propagation speed $U$ as a solution measure to explore this bifurcation, thus yielding the complementary bifurcation diagram for $h=0.05$ shown in figure 9.

We solve for the dimensionless tip speed $U$ as part of the system for steady finger propagation. However, as discussed in appendix B, we find from integrating over the domain that the tip speed $U$ is bounded below by a non-zero minimum $U_{\min }$ that is dependent on the particular channel height profile chosen; the minimum speed is 
achieved only when the finger fills the whole width of the channel, so that $y_{1,2}= \pm 1$. The non-zero minimum speed occurs due to our choice of non-dimensionalisation, which is based on the pressure gradient driving the flow.

When $h=0$, McLean \& Saffman (1981) showed that for $Q=0$, the finger fills the whole width of the channel and $U=U_{\min }=1$. The speed of the solution family found by McLean and Saffman is shown in figure 9 by the dash-dotted line. As $Q$ increases, the finger narrows monotonically and its speed increases, eventually reaching the halfwidth finger with $y_{1,2} \rightarrow \pm 1 / 2$ and $U \rightarrow 2$ as $Q \rightarrow \infty$.

For $h=0.05$, the minimum speed is $U_{\min }=0.967$ for the channel height profile we have chosen. At very low flow rates, the stable symmetric fingers are again very wide, and $U$ is close to $U_{\min }$. The propagation speed $U$ increases monotonically with $Q$ both for the stable part of the symmetric branch and for the asymmetric fingers. For all flow rates at this obstacle height, the stable branch is the one with the fastest propagation speed.

Figure 9 shows that there is a limit point on the symmetric branch at $Q=Q_{L S}>Q_{P}$, and so for $Q<Q_{L S}$ there are two different symmetric steady fingers for the same value of $Q$. The fingers directly connected to the pitchfork bifurcation are narrower and propagate faster than the second family of symmetric fingers. The second family of symmetric fingers is unstable to both symmetric and asymmetric perturbations, with the exchange of stability for symmetric perturbations occurring at the limit point $Q_{L S}$. We can track the location of the limit point $Q_{L S}$ as $h$ varies, as shown in figure 8 . Like the pitchfork bifurcation, the symmetric limit point $Q_{L S}$ moves to smaller $Q$ as $h$ increases, and persists as $h \rightarrow 0$ with $Q_{L S} \rightarrow \infty$. We have not found any interactions for $h>0$ between the pitchfork bifurcation $Q_{P}$ and the symmetric limit point $Q_{L S}$.

The finger shape on the symmetric branch evolves from round-nosed to doubletipped, through a smooth decrease from positive to negative lateral interfacial curvature measured at the point where the channel centre-line crosses the interface. The change in sign of the lateral curvature has no particular dynamical significance. In the case where $Q=0$, discussed in appendix $\mathrm{C}$, we calculate analytically the decrease of the centre-line curvature with increasing occluding height, and show that it changes sign without a bifurcation. Heil (1999) calculated a similar shape evolution, also without any bifurcation, for air fingers confined in buckled elastic tubes, under increasing tube compression. At the bifurcation points $Q_{P}$ and $Q_{L S}$, we find that the lateral centre-line curvature also decreases smoothly as $h$ is increased. The solution at $Q_{L S}$ becomes double-tipped for $h>0.046$ and the solution at $Q_{P}$ becomes double-tipped for $h>0.14$, as indicated by the crosses in figure 8 . The only stable symmetric fingers occur when $Q<Q_{P}$, and so at low heights only round-nosed fingers can be stable, but fingers with either shape may be stable for $h>0.14$.

Thus, there is at most one stable branch of symmetric steadily propagating fingers. The finger shape depends on the values of $Q$ and $h$, with double-tipped fingers found only for high enough obstacles. This is qualitatively consistent with experiments: both round-nosed and double-tipped steadily propagating fingers have been observed experimentally (e.g. figure $2(a, b)$ ) but never for the same control parameters, and the double-tipped fingers are observed for higher obstacles.

In our model, the channel height profile leads to spatial variations in transverse curvature in (2.9) (a surface-tension mechanism) and in mobility (a viscous mechanism), which affects the depth-averaged velocity in (2.7) and (2.8). We can test the influence of these two mechanisms by setting $b=1$ either in (2.9) or in (2.7) and (2.8) as appropriate, while retaining the height profile $b(y)$ in the other equations. Firstly, deactivating the transverse curvature mechanism, we obtain the 
same bifurcations for $h=0.05$, although these occur at larger $Q$, with $Q_{P}=0.084$ and $Q_{L S}=0.13$. As discussed in $\S 3.1$, at fixed $Q$, both mobility variations and transverse curvature variations will increase the velocity differential that drives symmetry breaking; when both mechanisms are active, we obtain asymmetric propagation modes over a greater range of $Q$ than when only one mechanism is present. This additive effect can explain why $Q_{P}$ increases when the transverse curvature mechanism is deactivated. If instead we retain only the transverse curvature mechanism, the bifurcation structure changes significantly; we do not find a limit point $Q_{L S}$ between symmetric branches, and thus the main symmetric branch exists for all $Q$, with at least one symmetry-breaking pitchfork bifurcation occurring on this branch, but the symmetric branch typically restabilises at large $Q$. These results suggest that the viscous mechanism underpins the steady bifurcation structure at low obstacle heights.

We also observe that $Q_{P}$ and $Q_{L S}$ increase significantly as $h$ decreases (see figure 8) and appear to tend to infinity as $h \rightarrow 0$. Examining (2.7)-(2.9) for small $h$, large $Q$ and fixed $\alpha$, we find that a distinguished limit occurs when $Q=O(1 / h)$. In this limit, the viscous corrections to (2.7) and (2.8) are of order $h$, and the contribution due to transverse curvature variation in (2.9) is of order $h / Q=O\left(h^{2}\right)$. This scaling confirms that the critical flow rates $Q_{P}$ and $Q_{L S}$ are determined by mobility variations for small $h$, while the effect of the variable transverse curvature is negligible.

Finally, the existence of multiple symmetric fingers of varying widths at the same $Q$ invites comparison to the results of Romero (1982) and Vanden-Broeck (1983), who found that a countably infinite family of fingers coexist with the solutions found by McLean \& Saffman (1981) in the case of $h=0$ and non-zero surface tension. A simple comparison can be made for the case where $Q=0.08$ and $\alpha=10$, corresponding to the inset shapes shown in figure 9 at $h=0.05$. For $h=0$ with $Q=0.08$ and $\alpha=10$, the numerical results presented by Romero (1982) indicate that the three narrowest solutions correspond to: (i) $\lambda=0.589$ and $U=1.70$, which is the Saffman-McLean finger shown in figure 6; (ii) $\lambda=0.899$ and $U=1.11$, which has a comparable velocity to the double-tipped finger with $U=1.14$ shown as an inset in figure 9; and (iii) $\lambda=0.982$ and $U=1.02$, which has much thinner liquid films for the same $Q$ than the solutions shown in figure 9. We have not found any evidence of interaction between the Romero solution branches and the multiple propagation modes in our work.

\subsection{Tip splitting}

The bifurcation diagram for $h=0.05$ shown in figure 9 indicates that there are no symmetric steadily propagating modes for $Q>Q_{L S}$ for the current formulation, which describes the propagation of a single air finger. In an experimental situation, if the finger shape is initially symmetric and the flow rate increased to a regime where no steady symmetric solutions exist, inherent asymmetries might be expected to trigger a switch to an asymmetric propagation mode. However, symmetric solutions must exist for $Q>Q_{L S}$, and we investigate these by solving in the half-domain and imposing symmetry conditions on the centre-line.

In order to investigate the solution behaviour in this regime, we perform timedependent calculations from the symmetric initial condition at the limit point, shown in figure $10(a)$. When $Q>Q_{L S}$ and symmetry conditions are enforced, we find that two narrow fingers emerge from the finger tip and eventually propagate steadily along the two sides of the channel. The two fingers are connected by a very slowly moving interface that crosses the channel centre-line. An instantaneous snapshot of this tip-splitting transition is shown in figure $10(b)$. 
(a)

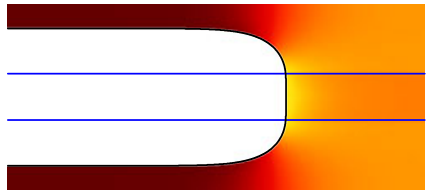

(c)

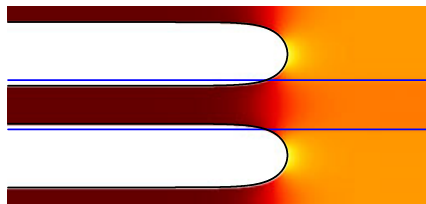

(b)

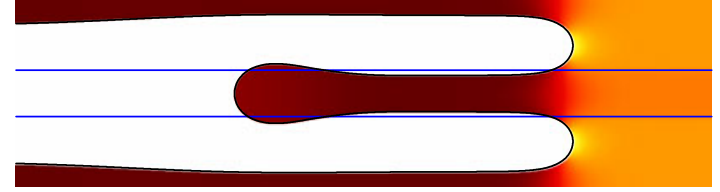

(d)

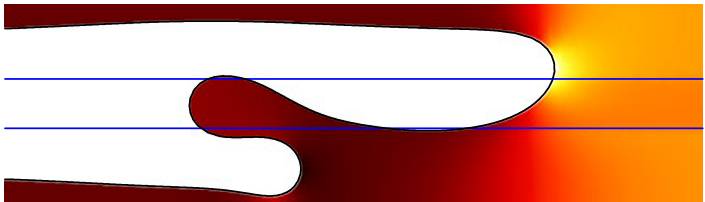

FIGURE 10. (Colour online) Finger behaviour near the symmetric limit point $Q_{L S}=0.099$ for $h=0.05$ : (a) finger shape (steady solution) at the limit point $Q=Q_{L S} ;(b)$ snapshot at $Q=0.12$ of the time-dependent calculation for $Q>Q_{L S}$ from the symmetric initial condition at the limit point, with imposed symmetry conditions, showing tip-splitting behaviour which transitions to a two-fingered state; $(c)$ two separate equal fingers obtained as symmetric copies of the steady solution of a reformulated system at $Q=0.12$, with the finger shape and speed agreeing well with the large-time behaviour of $(b) ;(d)$ snapshot at $Q=0.12$ of the time-dependent calculation for $Q>Q_{L S}$ from the symmetric initial condition at the limit point, but without imposed symmetry conditions, showing that the two-fingered state is unstable to asymmetric perturbations. The four images in this figure use a rescaled version of the standard scale shown in figure 6 to span the range from $u=-0.3$ to $u=1.9$. Both extrema in this range occur in $(d)$, where the dominant finger propagates with speed $U=1.89$ and the short stubby finger slowly retracts.

We can also reformulate the system to solve for the steady propagation of two separate symmetric fingers. In this case, the interface does not cross the channel centre-line and the symmetry conditions are exactly equivalent to the wall boundary conditions, so we simply solve for the propagation of one finger in half the channel width, with a non-symmetric depth profile. The resulting double-finger solution, shown in figure $10(c)$, agrees well with the calculated finger shape and speed from the long-time behaviour of the time-dependent solution.

The finger speed for the double-finger steady propagation mode is shown by the dotted line in figure 9. The double-finger mode always propagates more slowly than the asymmetric single fingers, but can propagate faster or slower than a single symmetric finger at the same flow rate $Q$. There is no continuous transition between double- and single-finger states within the realm of steady finger shapes, and so there is no exchange of stability.

Steady propagation modes with two equal fingers have not been reported in experimental investigations of the partially occluded rigid channel, although it is possible that they might be observed in experiments with suitable elimination of asymmetric perturbations. We investigated the stability of the double-finger state by repeating the calculation of figure $10(b)$ from the same symmetric initial condition but without the imposed symmetry conditions. The initial evolution again involves two fingers forming from the finger tip. However, the system is unstable to asymmetric perturbations induced by asymmetries in the mesh, and the interface eventually transitions to a single asymmetric finger shape, as demonstrated by the snapshot in figure $10(d)$. The instability of the double-fingered state with respect to asymmetric perturbations is consistent with the absence of states with two long 
fingers from experimental data for the rigid channel. We note that tip splitting and competition between fingers is a classic feature of experimental observations of fingering in Hele-Shaw cells at low surface tension (Park \& Homsy 1985; Maxworthy 1987; Tabeling et al. 1987) and in numerical investigations of evolution towards a single finger (Casademunt 2004). For these low-surface-tension cases, the tip-splitting behaviour is essentially triggered by experimental perturbations or numerical noise. However, for our model, the tip-splitting behaviour always occurs after the well-defined limit point $Q_{L S}$, while the transition from a double-finger state to a single-finger state through the preferential growth of one finger is again triggered by noise. We note that $Q_{L S}$ increases with decreasing $h$, indicating that tip splitting would be expected to be observed at low values of the surface tension for small obstacle heights.

The transition from a single finger for $Q<Q_{L S}$ to tip splitting for $Q>Q_{L S}$ can be explained by competition between geometry-induced mobility variation and the lateral curvature. For a given pressure gradient, the depth-averaged fluid velocity is proportional to $b^{2}$. As argued above, according to the kinematic boundary condition (2.8), the interface propagates more slowly in regions above the obstacle than in the deeper parts of the channel, i.e. there is a mobility-induced speed differential. In contrast, surface tension acts to hold the interface together, favouring steady propagation of a single finger. If the McLean \& Saffman parameter $B=\gamma /\left(3 \mu U^{*} \alpha^{2}\right)=1 /\left(3 \alpha^{2} U Q\right)$ is sufficiently large, steady propagation of a single finger can be achieved. However, for small $B$, the deformation associated with the mobility-induced speed differential overcomes the restoring effect of surface tension, and so tip splitting occurs. The speed differential increases with obstacle height for a given flow rate, requiring stronger surface tension to maintain a single finger, and so $Q_{L S}$ decreases as $h$ increases if $\alpha$ and $w$ are fixed.

\subsection{Transcritical bifurcation and localised fingers}

The final class of steadily propagating fingers in our model consists of narrow fingers that are localised to one side of the obstacle, such as the one shown in figure 11(a) for $h=0.4$. The localised fingers occur only for much larger obstacle heights than we considered previously and remain localised for very low $Q$, approximately for $Q<0.01$ at $h=0.4$. As $Q$ is increased, the finger spreads slightly over the obstacle but remains asymmetric, and there does not seem to be a continuous transition at fixed $h$ from the localised fingers to the symmetric solution branches.

The simplest picture consistent with our calculations is the occurrence of a transcritical bifurcation as illustrated in figure 12. This transcritical bifurcation connects the localised fingers to the asymmetric fingers arising from the symmetrybreaking bifurcation structure described in $\S 3.1$ for lower obstacle heights and occurs at a single obstacle height $h_{T}$. We conjecture that this transcritical bifurcation is also present in the experiments and is responsible for the different behaviours observed using wide and narrow obstacles shown in figure 3.

Evidence for a transcritical bifurcation also emerges from the bifurcation tracking results, where it appears as a turning point on the line tracking the limit point on the asymmetric branch, $Q_{L A}$ (see figure 8), yielding $h_{T}=0.123$. For $h<h_{T}$, a branch of localised fingers for $Q<Q_{L 3}$ should be stable, but these solutions cannot be reached by continuous evolution from the symmetry-breaking pitchfork. Examples of these isolated solutions are shown in figure 13. Unfortunately, we have been unable to explore the fate of the localised solutions as $Q$ decreases, because we cannot 
(a)

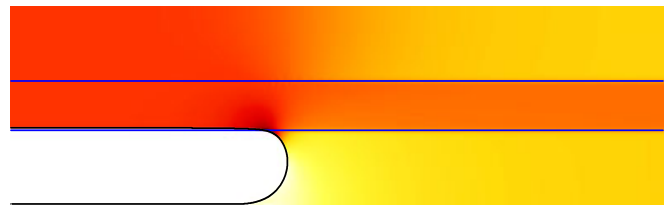

(b)

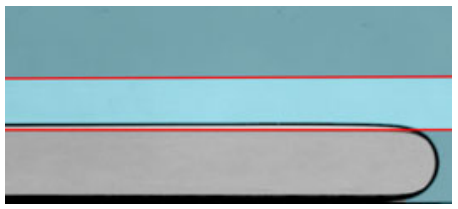

FIgURE 11. (Colour online) Comparison between numerical and experimental images of localised fingers. These propagate in the unoccluded region along one side of the obstacle. (a) Calculated finger shape for $h=0.4, Q=0.0061, w=0.25$ and $\alpha=10$ : here the finger speed $U$ is 2.10 , which is faster than the other fingers shown in this paper; we use a scale for this image with maximum value 2.3 and minimum value -1.8 ; the negative values of $u$ arise close to where the tip of the finger crosses the obstacle edge. $(b)$ Experimental image of localised finger propagation from figure 2 of Hazel et al. (2013), for $h=0.49$, $C a \equiv U Q \approx 0.003, w=0.25$ and $\alpha=6$.

(a)

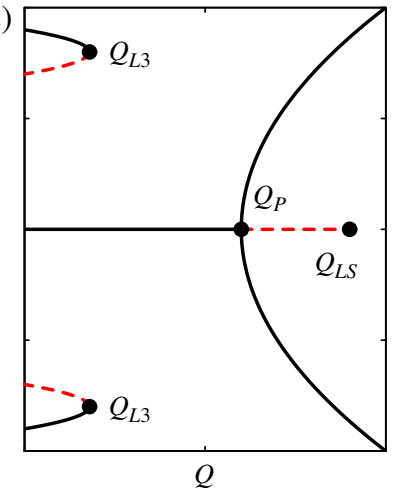

(b)

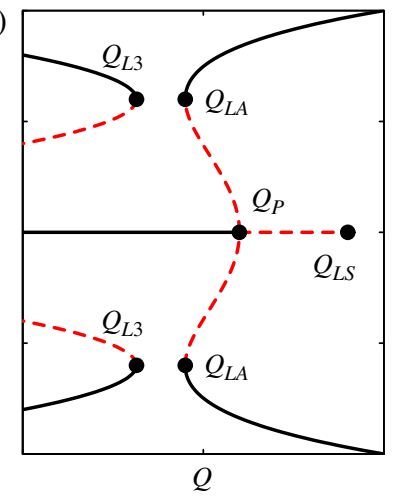

(c)

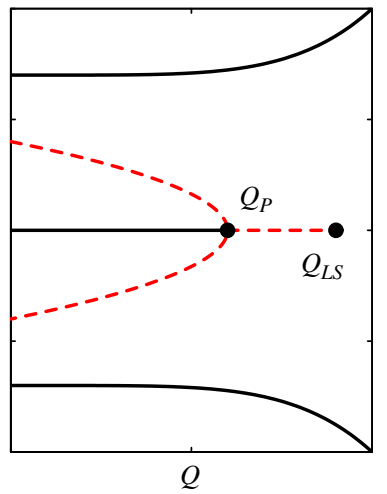

FIgURE 12. (Colour online) A sketch of a possible bifurcation picture involving a transcritical bifurcation at $h=h_{T} \approx 0.123$ between asymmetric steady solutions. This picture assumes the existence of two pairs of asymmetric solution branches for $Q<Q_{L 3}$. (a) At low $h$, the asymmetric solutions for large $Q$ are connected to the symmetric solutions via a supercritical pitchfork bifurcation at $Q=Q_{P}$, as shown in figure $7(a)$. (b) As $h$ increases, the pitchfork bifurcation transitions from supercritical to subcritical, as shown in figure $7(b)$, creating a pair of asymmetric limit points at $Q=Q_{L A}$. Subsequently, the limit point $Q_{L A}$ merges with another limit point $Q_{L 3}$ at a critical height $h_{T}$, where a transcritical bifurcation occurs. (c) For heights above $h_{T}$, the stable asymmetric solution branch is connected to localised fingers at low $Q$ and is disconnected from the symmetric branch.

resolve the extremely thin films that develop as the finger approaches one channel wall. This limitation prevents us from computing finger shapes close to the minimum propagation speed, as shown in figure 9, for example. It also makes it particularly problematic to explore the bifurcations at low $Q$ which occur between the subcritical pitchfork at $h=0.103$ and the localised asymmetric fingers at $h=0.4$. Thus, we cannot be certain that a more exotic sequence of bifurcations has not occurred.

Our most extensive calculations for obstacle heights above the putative transcritical bifurcation are for $h=0.15$, which has the bifurcation diagram shown in figure 14; this obstacle height is mainly of interest because of the existence of oscillatory propagation modes, which we discuss in the next section. We are able to compute a limited selection of asymmetric steady solutions very near the pitchfork bifurcation, 
(a)

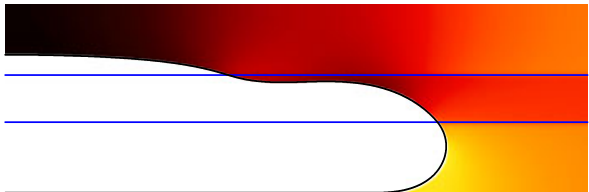

(b)

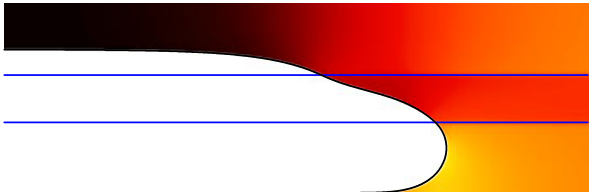

Figure 13. (Colour online) Two solutions on the $Q<Q_{L 3}$ branch segment shown in figure 12(b); the connections between the pair shown here and the two asymmetric solutions shown in figure $7(b)$ change at the transcritical bifurcation. (a) Finger at $h=$ 0.123, $Q=7.5 \times 10^{-3}$ and $U=1.306$; this finger connects to the stable asymmetric branch sketched in figure 12(c), with interface shapes for larger $h$ shown in figure 11. (b) Finger at $h=0.123, Q=0.0102$ and $U=1.264$; this finger connects to the unstable asymmetric branch that arises from the pitchfork.

(a)

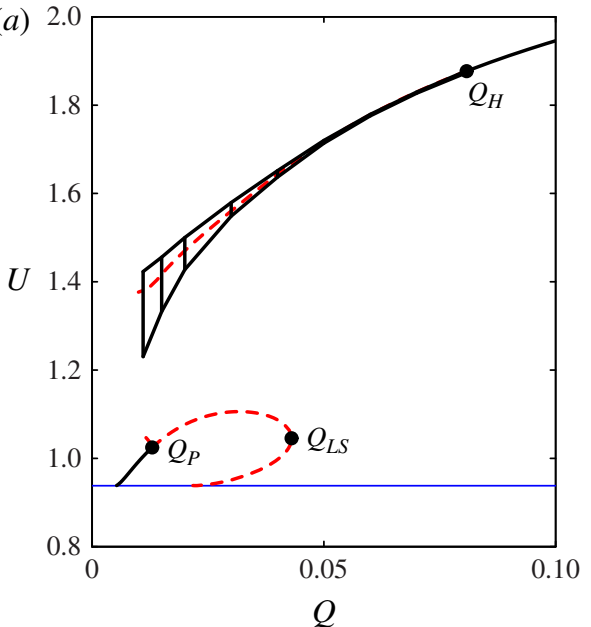

(b)

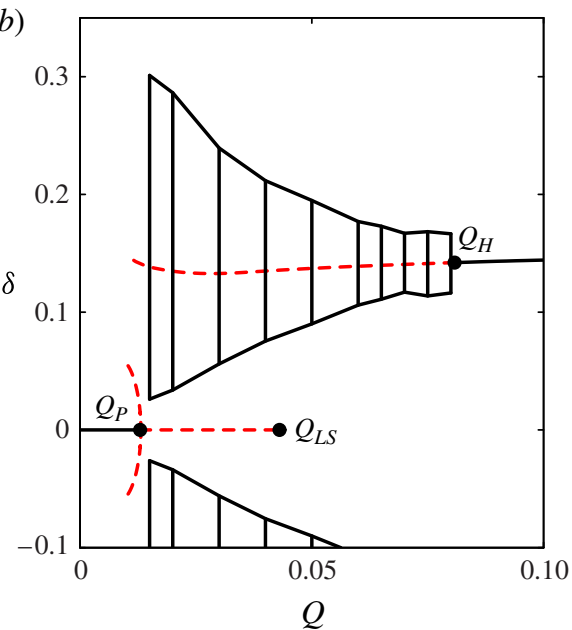

FIGURE 14. (Colour online) Bifurcation diagram for steady and oscillatory finger propagation modes for $h=0.15:(a)$ the speed $U ;(b)$ the offset $\delta$. The pitchfork bifurcation at $Q=Q_{P}$ is subcritical for this obstacle height. We can calculate only a very short branch of asymmetric solutions connected to the pitchfork. The oscillatory solutions are shown by the black vertical lines and associated envelope. We find that the oscillatory modes surround the most asymmetric branch of steady solutions. The oscillation amplitude decreases as $Q$ increases, and the oscillations vanish at a Hopf bifurcation at $Q=Q_{H}=$ 0.081 .

and also a branch of asymmetric steady solutions for larger $Q$. However, these two segments of asymmetric branches do not appear to be connected, which is consistent with the branch connections sketched in figure 12(c) for $h>h_{T}$.

Localised fingers exist only for very small $Q$, so we now make a comparison with results in the $Q \rightarrow 0$ limit for our depth-averaged model. At $Q=0$, we find from (2.9) that the total curvature of the finger interface is constant, with no effect from the pressure variations within the fluid, and we can solve a system of coupled ordinary differential equations to determine the interface shape, as discussed in appendix $\mathrm{C}$. We find that the single-finger interface shapes for $Q=0$ are of two types: (i) symmetric fingers, filling the whole width of the channel, which exist only for $h<h_{\max }=0.40$; or (ii) asymmetric localised fingers, which exist only for $h>h_{\min }=0.21$. 
The appearance of localised fingers is another important area of qualitative agreement between our depth-averaged model and the experimental system. Typical shapes of localised fingers in the two systems are shown for comparison in figure 11. In both cases, localised fingers are observed at low flow rates, and occur as part of broadly similar bifurcation sequences.

\section{Oscillatory propagation modes in the depth-averaged model}

\subsection{Characterisation of oscillatory propagation modes}

A striking feature of the experimental observations of finger propagation is the spontaneous emergence of oscillatory states. Here 'oscillatory' means that the moving finger tip leaves behind a spatially varying periodic disturbance which then remains stationary in the laboratory frame. An instantaneous experimental image of the finger shape is shown in figure $2(d)$.

As discussed in $\S 1$, Pailha et al. (2012) proposed a surface tension-based mechanism for the oscillations, centred on the sudden decrease in transverse curvature as the interface passes over the edge of the obstacle. This decrease in finger curvature raises the fluid pressure just outside the interface, which drives a fluid flow away from the interface, resulting in the rapid sideways motion of the interface away from the obstacle. Eventually the expanding interface is constrained by the channel side-walls, providing the return mechanism. If the mechanism is correct, then the oscillatory propagation modes require both transverse curvature variation and a finger configuration where the interface can expand over the edge of the obstacle. Our depth-averaged model allows us to test these assumptions.

We find that we can induce previously unseen oscillations about a symmetric finger in our model, in a region far removed from bifurcations between steady solutions. We take an obstacle that has $h=0.05$ but is very wide, with $w=0.75$, so that the symmetric finger must pass over the edge of the obstacle at a finite value of $Q$ as it transitions from occupying the full channel width at very low $Q$ to half the channel width at $Q=\infty$. The two sides of the finger are almost parallel to the edge of the smoothed obstacle. The symmetric finger becomes unstable when the finger edge is very near the smoothed edge of the obstacle, developing an oscillatory instability as shown in figure 15. The interface evolves with time, with the shape of the tip quickly settling into periodic behaviour. In time-dependent simulations, the two edges of the finger pass over the edges of the obstacle simultaneously, and here the base solution is unstable to two pairs of eigenmodes, such that the oscillations may occur as sinusoidal or varicose displacements, as shown in figure 16. More generally, however, either side of the finger can oscillate independently of the other. We test the effect on the oscillatory instability of the transverse curvature and variable mobility by deactivating each mechanism in turn. The results are shown in figure 15: the oscillations occur only when the edges of the finger are close to, and parallel to, $y= \pm w$, and we find that the oscillatory instability occurs only when transverse curvature is included, which confirms the driving mechanism proposed by Pailha et al. (2012).

For narrower obstacles, symmetric oscillations would require the edges of the symmetric finger to be near the obstacle boundary, which is not possible if $w<1 / 2$. Instead, the oscillatory modes are asymmetric and only one side of the finger is close to the obstacle, while the other is close to the channel wall. A selection of instantaneous interface shapes is shown in figure 17 for $w=0.25, \alpha=10$ and $h=0.15$. These oscillatory propagation modes are obtained in our model by time integration from a smooth, asymmetric, non-equilibrium finger shape. We work in a frame that 


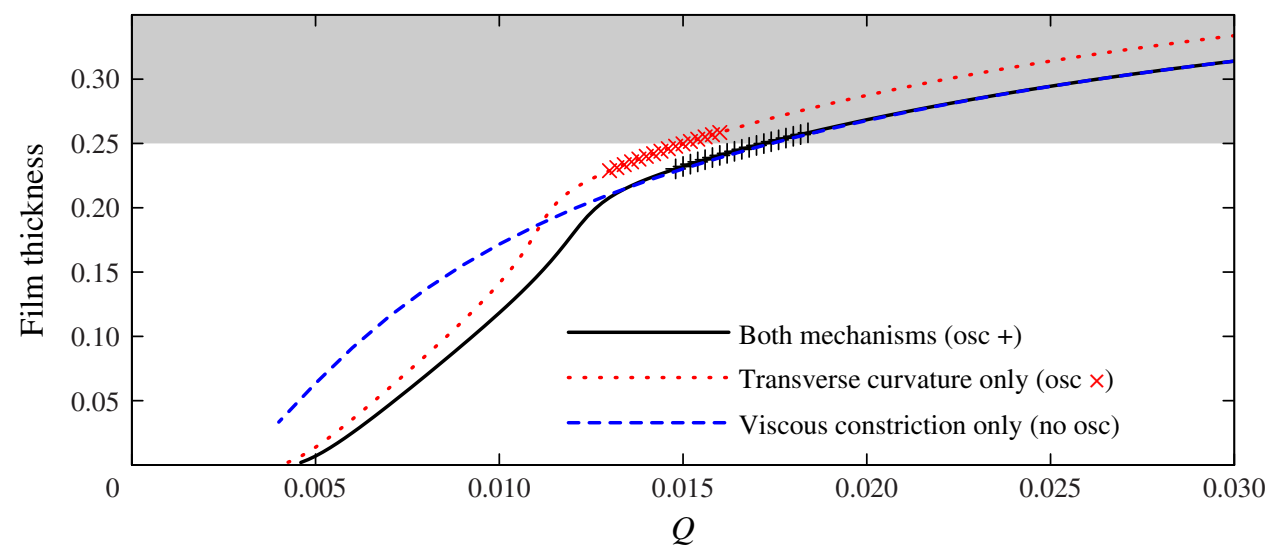

FIGURE 15. (Colour online) Film thickness $1-y_{2}$ (see figure 5) and linear stability properties as a function of flow rate $Q$ for $h=0.05, w=0.75$ and $\alpha=10$. In this case, the finger shapes are perturbations to the unoccluded shape with finite surface tension. The inclusion or otherwise of transverse curvature variations has no effect on the shape or speed of steadily propagating fingers that are narrower than the obstacle width. For both cases where the variable transverse curvature terms are included, the steadily propagating finger is unstable to oscillatory instabilities when $y_{2} \approx w$, i.e. when the edge of the finger is close to the smoothed edge of the obstacle. No oscillatory instabilities occur when the transverse curvature mechanism is neglected.

FIGURE 16. (Colour online) Instantaneous snapshot of oscillation of symmetric base state for $Q=0.016, h=0.05$ and $w=0.75$. Here the oscillation appears to be varicose, but the linear stability analysis also reveals instability to a sinusoidal perturbation with the same eigenvalue.

is always moving instantaneously with the finger tip at speed $U$; in this frame the waves propagate away from the finger tip and are observed to grow in amplitude as they do so. The finger speed $U(t)$ varies over the period of oscillation, and is plotted for a variety of oscillatory states in figure 18 .

For each propagation mode shown in figure 17, the interface is oscillatory only along the edge of the finger that is closest to the edge of the obstacle at $y=w$. As for the case illustrated in figure 16, the oscillations are induced when the interface is standing on top of and parallel to the obstacle edge at $y=w$. The other side of the finger lies close to the wall on the side of the channel, and this edge of the finger remains straight. The finger tip thus spans the region over the obstacle and one of the two unoccluded regions.

For $h=0.15$, we are able to obtain oscillatory propagation modes for $Q$ in the range 0.011-0.081. At the lowest $Q$ in this range, the oscillations first appear near the finger tip, and the relative amplitude of finger speed variation over the period of an oscillation is up to $10 \%$. As $Q$ increases, the oscillations first appear further behind the finger tip, and there is a significant reduction in the variation of the tip 

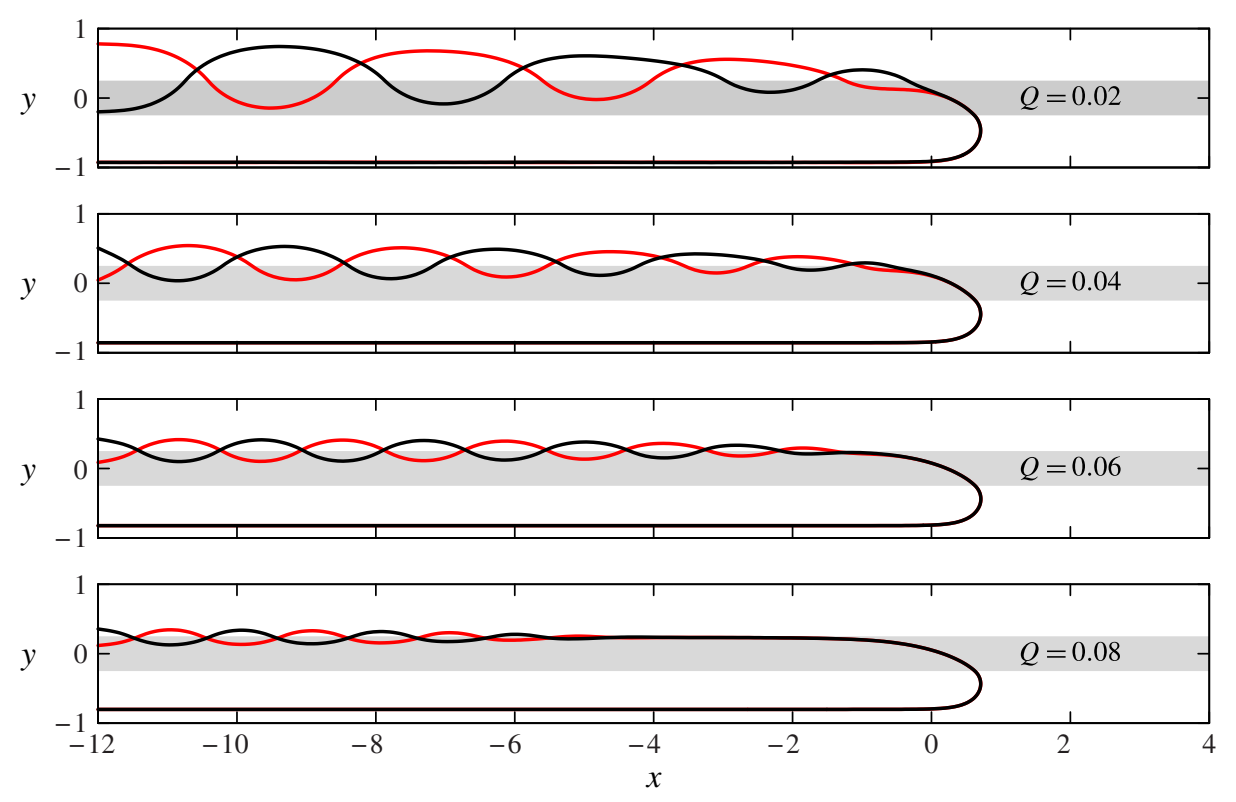

FIgURE 17. (Colour online) Instantaneous interface shape for oscillatory propagation modes at $h=0.15$, with $Q$ as shown. In each case, the two instants shown are for the maximum and minimum interface displacement at the truncation point $x=-12$. The base solution and least-stable eigenmode pair for the corresponding asymmetric steady propagation modes are shown for comparison in figure 19.

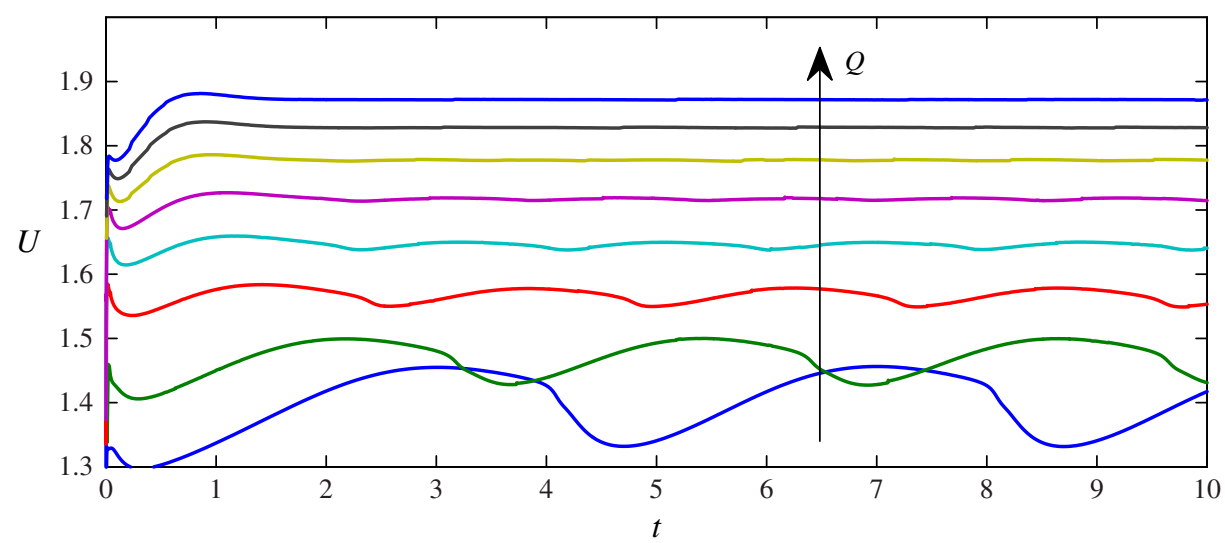

FIGURE 18. (Colour online) Finger propagation speed $U(t)$ for oscillatory propagation modes with $h=0.15$ and $Q=0.015,0.02,0.03,0.04,0.05,0.06,0.07,0.08$. For this solution family, the amplitude of oscillations in $U$ decreases, and the frequency increases as $Q$ increases. The maximum and minimum values of finger offset $\delta$ and speed $U$ over a period of oscillation once initial transients have decayed are shown in figure 14; the orbits appear to surround the steady asymmetric branch.

shape and the speed $U$ over the period of an oscillation. The spatial wavelength and oscillation time period also decrease as $Q$ increases. Eventually, by $Q=0.081$, the waves no longer appear within the finite domain used for our calculations, and the 
system appears to restabilise towards an asymmetric state. However, the decay of these oscillations as they leave the domain is sensitive to end effects and is difficult to resolve consistently, as demonstrated by the irregular amplitudes for the finger offset $\delta$ near $Q=0.081$ shown in figure 14 .

The calculated oscillatory modes shown in figure 17 have many similarities to the experimentally observed states shown in figure $2(d)$, which were explored extensively by Pailha et al. (2012). In both cases, the finger shapes are asymmetric, oscillations are only visible far enough behind the tip that the interface has reached the line $y=w$, and there are no discernible periodic disturbances to the tip shape. As $Q$ is increased for a channel with fixed cross-section, waves are shed progressively further behind the finger tip, and the wavelength of the oscillations decreases.

In experiments, the finger tip shape does not change measurably during a period of oscillation and the finger tip speed is observed to remain constant within the $2 \%$ experimental error (Pailha et al. 2012) for all reported experimental states. In our model, the finger tip speed variation over a period can be up to $10 \%$ at the lowest values of $Q$, but the corresponding finger shapes are quite different from those observed in experiments because the oscillation begins very near the finger tip; see figure 17. Propagation modes similar to the experimental ones occur in our model at larger $Q$ where there is no noticeable variation in $U$ over the period of these oscillations, in accord with the experiments.

Many of the reported experimental oscillatory modes are for higher obstacles and lower $Q$ than we have considered here. For large-amplitude oscillations in this range, the interface shape is no longer sinusoidal in nature, but instead more resembles a square wave, with the interface limited by the sharp step and the channel walls (e.g. figure 3 of Pailha et al. 2012). The assumptions underlying our model break down in such cases, which feature complex three-dimensional effects, particularly near the channel side-walls. We do not attempt to replicate this behaviour in our model.

\subsection{Oscillatory modes and the bifurcation structure}

We now return to figure 14, which shows the resulting bifurcation diagram for $h=0.15, w=0.25$ and $\alpha=10$. We use the two measures $U$ and $\delta$ to characterise the oscillatory propagation modes and to enable comparison with our bifurcation structure for steady propagation modes. We find that the oscillations surround the most asymmetric of the steadily propagating solution branches. Without loss of generality, we shall now limit our discussion to steady and oscillatory fingers where the tip of the finger has $y<0$, such as the cases shown in figure 17. As the oscillatory propagation modes are unsteady, we define $U$ as the frame speed required to prevent instantaneous translation of the finger tip in the $x$ direction, which is a property of the tip shape and should be largely independent of domain truncation. We define $\delta=\left(y_{1}+y_{2}\right) / 4$, where $y_{2}$ varies significantly over the period of oscillation but $y_{1}$ does not. As shown in figure 17, the wave amplitude grows with distance from the finger tip, and so there is growth in the amplitude of $y_{2}$, and hence $\delta$, depending on the distance behind the finger tip that this displacement is measured. For convenience, we have chosen to measure $y_{2}$ at the truncation point; however, this choice may also cause $\delta$ to be sensitive to local effects of the truncation boundary conditions.

Given that the oscillations surround the most asymmetric branch of steady solutions, we now use a linear stability analysis to investigate the stability properties of this branch. For an obstacle height of $h=0.15$, our calculations show that the finiteamplitude oscillatory propagation modes exist whenever the corresponding asymmetric 

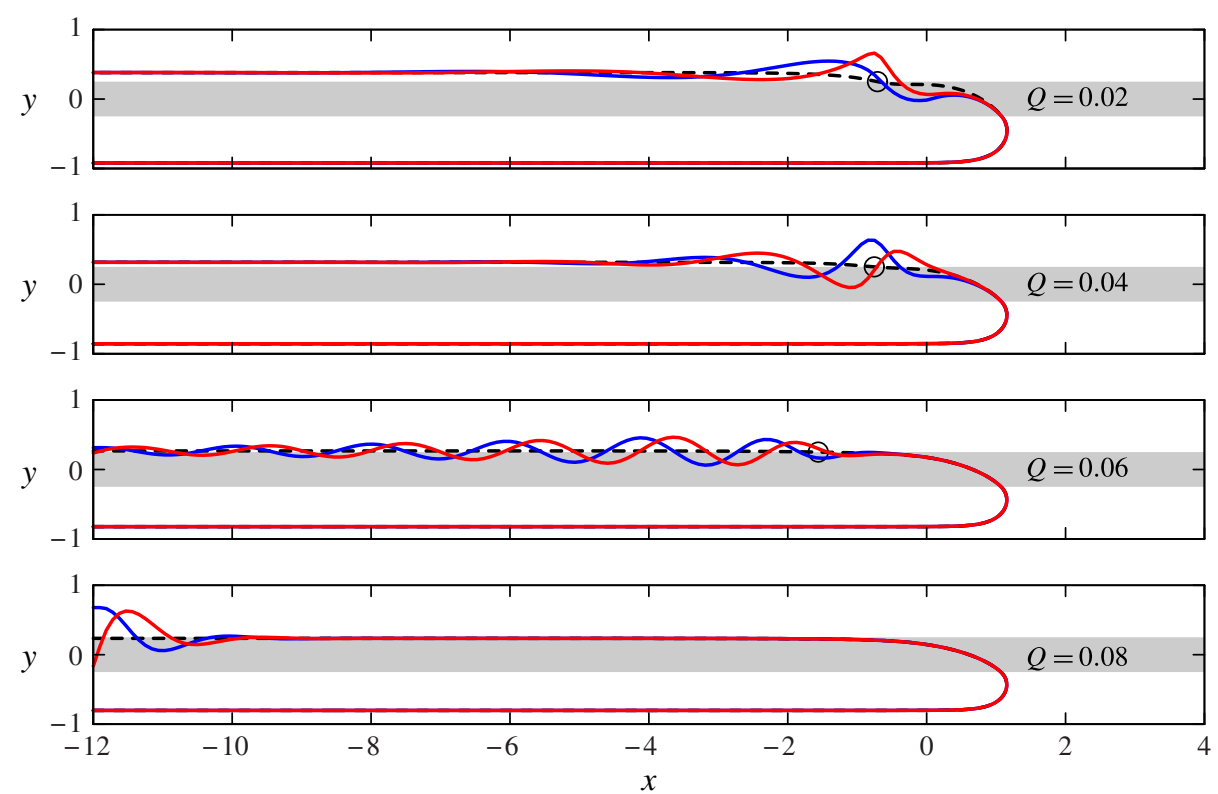

FIGURE 19. (Colour online) Base solution (dashed line) and the least-stable complexconjugate eigenvector pair (solid lines) for the same parameters as the finite-amplitude oscillatory solutions shown in figure 17. The corresponding eigenvalues are $\lambda=0.777 \pm$ $1.85 \mathrm{i}, \lambda=1.14 \pm 4.08 \mathrm{i}, \lambda=2.20 \pm 5.83 \mathrm{i}$ and $\lambda=0.420 \pm 5.91 \mathrm{i}$, so in each case the base solution is unstable to the eigenvector pair shown. These are the only unstable eigenmodes, except in the $Q=0.06$ case, where the base solution is unstable to at least five complex-conjugate eigenvector pairs. The circles mark the points where the interface for the base solution crosses the line $y=w$.

steady solution is unstable to one or more complex-conjugate eigenmode pairs. The spatial structure of the most-unstable eigenmodes mimics the spatial structure of the finite-amplitude oscillations, with corresponding interface displacements shown in figure 17 for time-dependent calculations and figure 19 for the linear stability calculations. We have verified that the time period for oscillations predicted by the linear stability analysis is comparable to that exhibited by the finite-amplitude oscillations. The eigenmodes shown in figure 19 represent the initial growth of the instabilities, which occurs very close to the finger tip at low $Q$ and moves progressively further from the finger tip as $Q$ increases. The interface displacement far away from these triggering regions is due to the movement of the finger leaving behind an oscillatory disturbance which then grows slowly in the laboratory frame.

The computations for low $Q$ at $h=0.15$ for both the finite-amplitude oscillations and the asymmetric steady solutions are limited by difficulties in resolving the thin film and associated transition region along one side of the finger. We are unable to determine the fate of either the limit cycle or the asymmetric steady solution branch as $Q \rightarrow 0$; the extent of our computations is illustrated in figure 14 for $h=0.15$. We note that this obstacle height is above the transcritical bifurcation at $h=h_{T}=0.123$ but significantly below the heights at which truly localised fingers occur.

As $Q$ increases, the oscillation amplitude decreases, and the linear stability calculations confirm that the oscillatory propagation modes disappear through a Hopf bifurcation at $Q=Q_{H} \approx 0.081$, where the asymmetric steady solution branch 


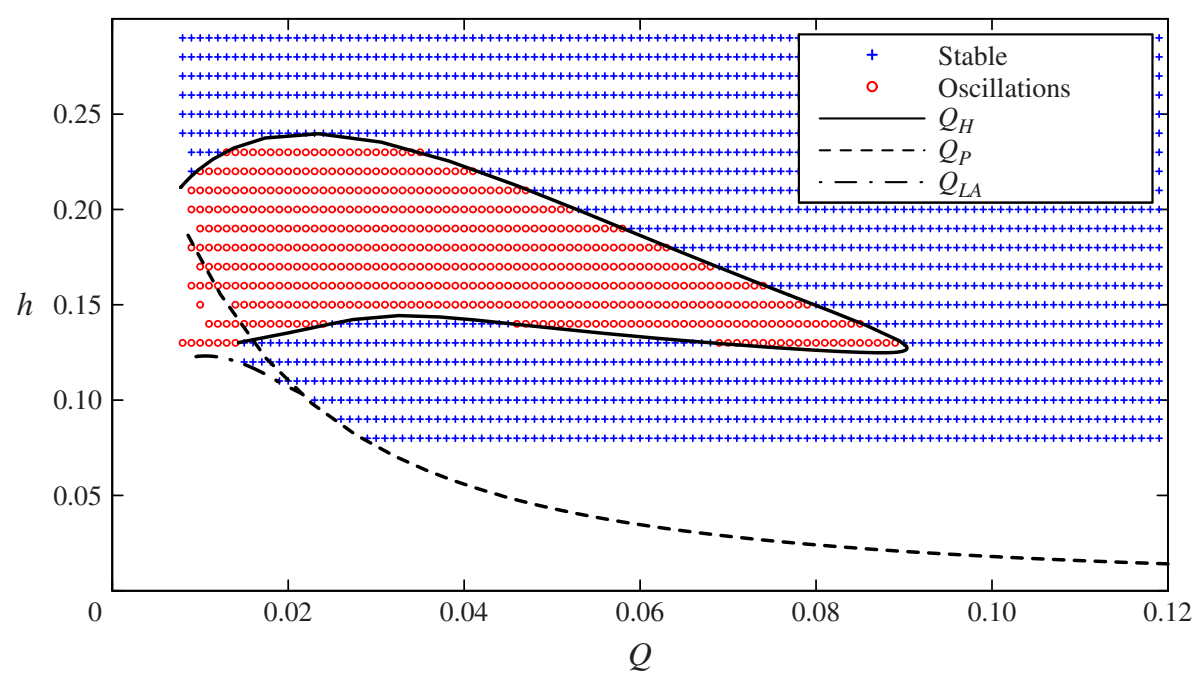

FIGURE 20. (Colour online) The bifurcation diagram from figure 8 with the addition of linear stability information for steady propagation modes on the most asymmetric branch. For simplicity, we have not shown the symmetric limit point $Q_{L S}$ as it does not interact with the other bifurcations shown here. Circles indicate solutions unstable to at least one complex-conjugate pair of eigenmodes, while crosses indicate stable asymmetric solutions. The solid line is the result of a Hopf bifurcation tracking calculation of a single complex-conjugate eigenvalue pair, and correctly predicts the stability boundary for oscillatory propagation modes.

regains stability. The interface displacement for both the finite-amplitude oscillations and the unstable eigenmodes is localised near the domain truncation point when $Q \approx Q_{H}$, and the calculations are sensitive to truncation effects. We find that inclusion of the alternative boundary condition (2.6) used for the time-dependent calculations has little effect on the stability boundary. We also explore the effect of the truncation conditions by extending the domain length; we find that this results in a shift of the Hopf bifurcation to a slightly larger value of $Q$, but it does not alter the interface shape or the form of the bifurcation. For an infinite channel, oscillations should occur only when the finger edge is very near the smoothed edge of the obstacle, which is the case over a finite range of $Q$, and so $Q_{H}$ remains bounded.

The linear stability calculations allow us to investigate oscillatory behaviour as $Q$ and $h$ vary by analysing the stability properties of solutions along the steady asymmetric branch. We first calculate the 10 least-stable eigenmodes at a large number of values of $Q$ and $h$, with results shown by the symbols in figure 20 . We find that oscillatory instabilities occur in a bounded region of parameter space and restabilise completely for sufficiently high obstacles.

For very low obstacle heights, the asymmetric finger shapes are stable whenever they exist. As $h$ is slowly increased, we find that oscillations arise in two disjoint ranges of $Q$. Oscillatory instabilities of the finger tail first appear at $h=0.125$ and $Q=0.088$. Oscillatory instabilities of the finger tip arise at similar parameters to the transcritical bifurcation at $h=0.123$ and $Q=0.0106$, but detailed investigation shows that the stability boundary does not coincide exactly with the transcritical bifurcation point. As $h$ increases further, the two regions of instability merge into a single region, which shrinks and eventually vanishes by $h \approx 0.24$, so that the asymmetric solution 
branch is always stable for $h>0.24$. For $0.21<h<0.24$, we find that the oscillations are bounded in $Q$ by a pair of Hopf bifurcations. However, it is not clear how or whether the oscillations restabilise at low $Q$ for lower heights, i.e. for $0.123<h<0.21$, given that the computations at low $Q$ in this region are limited by thin-film effects in our numerics, as discussed in $§ 3.4$.

As well as the linear stability analysis, we can use a bifurcation tracking algorithm to explicitly track a Hopf bifurcation as both $Q$ and $h$ vary, which correctly predicts the boundary of the oscillatory region, as shown by the solid line in figure 20. For $h=0.15$, we find that as $Q$ varies there is a continuous transition in the least-stable eigenmode, with spatial structure shown in figure 19, although a large number of other eigenmodes are also unstable within this region near $Q=0.06$. We note that the Hopf tracking algorithm involves conditions on a single complex-conjugate eigenmode pair, and so the agreement between the Hopf bifurcation $Q_{H}$ shown in figure 20 with the stability boundaries obtained through linear stability analysis provides strong evidence that the instabilities of the tip and tail occur as a result of a single continuously varying eigenmode.

We have conducted a similar linear stability analysis as $Q$ and $h$ vary for an alternative channel with $\alpha=4.5$ and $w=0.33$; the cross-section for this channel is shown in figure 4(b). We obtain a qualitatively similar bifurcation diagram, with a supercritical pitchfork at low $h$ transitioning to subcritical at $h=0.15$ and $Q=0.10$, and what appears to be a transcritical bifurcation at $h=0.22$ and $Q=0.055$, again at the lower limit of tractable $Q$ due to thin-film effects. The oscillatory instability of the tip again arises near the transcritical bifurcation, but the tail becomes unstable at $h=0.145$ and $Q=0.29$, confirming that there is no need for the tail instability to occur at a similar height to the transcritical bifurcation.

Pailha et al. (2012) explored the bifurcation structure for oscillatory propagation modes within the experimental system. While the bifurcation structure they present has a number of broad similarities to our model system, we should not necessarily expect the two bifurcation structures to be related. In both experiments and our model, the oscillations disappear as $Q$ increases through a Hopf bifurcation which restabilises an asymmetric solution branch. The agreement between the two bifurcation structures at low $Q$ is less clear.

For small $Q$, the experimental investigations suggest that the oscillatory propagation modes are lost through a homoclinic bifurcation at $C a=U Q=C a_{c 1}$, using the notation of Pailha et al. (2012), in which the limit cycle collides with an unstable steady propagation mode. This hypothesis is supported by observations of a logarithmic divergence in the spatial wavelength of oscillations near the critical capillary number $C a_{c 1}$; oscillatory states are observed only for $C a>C a_{c 1}$. For $C a<C a_{c 1}$ the system switches to a symmetric steady propagation mode. As $Q$ decreases for oscillatory states in our model, we observe an increase in the spatial wavelength in a direction consistent with the critical slowing-down described by Pailha et al., but we do not have sufficient evidence to support statements regarding logarithmic divergence. Despite considerable effort, we have been unable to complete either time-dependent or steady asymmetric calculations at very low $Q$; it is possible that a variety of bifurcations might occur in our model below the flow rates at which we are able to compute solutions.

\section{The effect of obstacle smoothing on linear stability of a straight interface}

The instability of the wide finger discussed in $\$ 4.1$ suggests that the interface becomes unstable to oscillations when one or both of the sides of the finger passes 
over the edge of the obstacle. We can model the side of the finger as an infinite straight interface aligned parallel to the channel side-walls, and thus estimate the wavelength of the oscillation. A straight interface at $y=Y_{0}$ can be at rest provided that the pressure inside the fluid domain is equal to $p_{b}-1 /\left(3 \alpha Q b\left(Y_{0}\right)\right)$. We now consider the time-dependent linearised evolution of a small disturbance to this straight interface, of the form

$$
Y(x, t)=Y_{0}+\eta_{0} \mathrm{e}^{\lambda t} \sin k x,
$$

where the fluid occupies the region $Y(x, t)<y<1$. We will assume that $\eta_{0} / Y_{0} \ll 1$ and that the interface extends to $-\infty<x<\infty$. As discussed in $\S 4.1$ and illustrated in figure 15, the transverse curvature mechanism is responsible for the emergence of oscillatory propagation modes. For convenience, we now neglect the viscous mechanism, so that $b=1$ in (2.7) and (2.8), but retain the transverse curvature variation. We apply the linearised forms of (2.7)-(2.10) and solve for $\lambda(k)$, assuming that the perturbation pressure is proportional to $\sin k x$, thus obtaining

$$
\lambda(k)=\frac{1}{3 \alpha Q} k \tanh \left[k\left(1-Y_{0}\right)\right]\left(\frac{b^{\prime}\left(Y_{0}\right)}{b^{2}}-\frac{k^{2}}{\alpha}\right) .
$$

If $b^{\prime}\left(Y_{0}\right)<0$, the interface is always stable. However, for both the symmetric and the asymmetric oscillatory states discussed in $\S 4$, we find that $b^{\prime}\left(Y_{0}\right)>0$ and $Y_{0} \approx w$. In this case, we find that there is a finite $k_{\max }$ corresponding to the maximum positive growth rate in (5.2); waves at sufficiently high wavenumber will decay. For large $k$, we can set $\tanh k\left(1-Y_{0}\right)=1$ in (5.2) and evaluate $b^{\prime}\left(Y_{0}\right)$ from (2.1) to obtain

$$
k_{\max }^{2}=\frac{\alpha b^{\prime}\left(Y_{0}\right)}{3 b^{2}} \approx \frac{\alpha h s}{6(1-h)^{2}},
$$

where the parameter $s$ controls the smoothing of the step, as illustrated in figure 4 . For both (5.2) and (5.3), we find that the critical wavenumber and the corresponding growth rate are dependent on the channel slope; $b^{\prime}\left(Y_{0}\right)=h s / 2$ at the point of maximum slope, and so for a given obstacle height, the wavelength for oscillations is controlled by the sharpness parameter $s$ and scales as $O\left(s^{-1 / 2}\right)$.

The stability analysis for a uniform straight interface predicts that the wavelength of the least-stable perturbations is dependent on the sharpness parameter $s$ via (5.3). However, the interface of a steadily propagating finger is never straight, and so the stability thresholds may be affected by the finger shape. We use the wide channel oscillation to study the effect of varying $s$ on the linear stability for oscillatory finger propagation modes. We set $h=0.05$ and $w=0.75$, and choose $Q(s)$ so that $y_{1,2}= \pm w$ and the edge of the steadily propagating finger is always exactly aligned with the steepest part of the obstacle. We use our numerical linear stability analysis, as discussed in appendix A, to calculate eigenvalues for fingers similar to the one shown in figure 16. We find that the growth rate of the most-unstable eigenmode has a strong dependence on $s$ and has a similar scaling for large $s$ to the predicted maximum growth rate $\max \{\lambda(k)\}$ from the idealised expression (5.2). However, all of the eigenmodes grow more slowly than (5.2) would suggest; indeed, all are stable when $s<26$. Thus, the tip dynamics and domain truncation, included in the full system for finger propagation, have a stabilising influence on oscillatory disturbances in comparison to the behaviour of an infinite straight interface, but the wavelength 
is still dominated by the obstacle smoothing via the transverse curvature variation in the dynamic boundary condition.

In the experiments, oscillations occur with a regular wavelength even for a sharp step with no obvious obstacle smoothing beyond the small-scale imperfections resulting from milling of the obstacle. The depth-averaging assumptions break down in the case of a sudden height variation, and in particular the fluid films underneath the air finger are likely to be thicker in the corner just next to the step. These fluid films might create some effective smoothing regarding the rate of transverse curvature variation as the finger moves over the step. However, full three-dimensional calculations would be required to resolve the interface shape and drainage of the fluid film, in order to establish how the wavelength is set for oscillations in the case of a sharp step.

\section{Discussion}

In this paper, we have derived a depth-averaged model to describe the propagation of semi-infinite air fingers into wide fluid-filled channels with partially occluded cross-sections. We chose a smoothed centred step-like occlusion profile to mimic the experiments of de Lózar et al. (2009), Pailha et al. (2012) and Hazel et al. (2013). We solved our model numerically using a finite element discretisation, and conducted a detailed analysis of bifurcations in this model for a particular channel profile as the height of the occlusion is increased from zero.

Our model is able to reproduce all the essential qualitative features of the experimental results. Multiple finger propagation modes can coexist at the same control parameter values, even for very low obstacles. The finger propagation modes we find include asymmetric and symmetric steady propagation modes, as well as oscillatory propagation modes, each of which resembles states observed experimentally. We also found a new mode of time-dependent finger behaviour involving tip splitting, and a corresponding steady propagation mode involving two separate fingers. We now summarise the changes in behaviour as the obstacle height $h$ is increased from zero.

There is a robust bifurcation structure for low obstacle heights. At very low flow rates, the finger is symmetric and strongly resembles the unoccluded finger shape. As $Q$ increases, this finger loses stability through a supercritical symmetry-breaking pitchfork bifurcation at $Q=Q_{P}$. The asymmetric branches which emerge through this pitchfork are stable for all $Q>Q_{P}$. Continuing the symmetric branch beyond the pitchfork bifurcation, we find that the symmetric branch merges with a second branch of symmetric solutions at a limit point $Q_{L S}$. All solutions on the second symmetric branch are unstable.

Bifurcations between steady symmetric solutions beyond the pitchfork bifurcation cannot be investigated experimentally as they are unstable to asymmetric perturbations. However, we used our model to explore finger behaviour in a perfectly symmetric system and to investigate the behaviour of symmetric fingers beyond the limit point $Q_{L S}$ where symmetric solutions for steady propagation of a single finger disappear. In this regime, time-dependent simulations of finger propagation show tip-splitting behaviour, in which two narrow fingers are observed to emanate from the finger tip and propagate along the two sides of the obstacle. The speed of these narrow fingers is equal to that for a reformulated system with two distinct fingers in the same channel. These double-fingered and tip-splitting modes are unstable to asymmetric perturbations in our model through the preferential growth of one of the two fingers, which then expands to become a single asymmetric finger. Tip-splitting behaviour and subsequent 
finger competition are ubiquitous phenomena in Saffman-Taylor fingering for systems with very low values of the parameter $B=\gamma /\left(3 \mu U^{*} \alpha^{2}\right)=1 /\left(3 \alpha^{2} U Q\right)$; in contrast, we find that these behaviours occur in a regime with much larger $B$. In fact, the doublefingered (two disjoint interfaces) and double-tipped (single connected interface) states that we observe in our model are intriguingly similar to states with two long fingers observed during experimental investigations of airway reopening, in which the channel walls are elastic and the channel is largely collapsed ahead of the propagating finger (Heap \& Juel 2008). This suggests that channel elasticity and deformation may act to suppress symmetry-breaking instabilities. We note that for the system investigated experimentally by Heap \& Juel, there is a decrease in channel height ahead of the finger due to the initial collapsed state of the channel, giving a taper in channel height that has a stabilising effect on viscous fingering (Al-Housseiny, Tsai \& Stone 2012; Pihler-Puzović et al. 2012).

We tracked the pitchfork bifurcation $Q_{P}$ and symmetric limit point $Q_{L S}$, and found that both move towards lower $Q$ as $h$ increases. The first significant change is that the pitchfork bifurcation transitions from supercritical to subcritical, which would lead to hysteresis in steady propagation modes. We note that both supercritical and subcritical symmetry-breaking bifurcations have been reported for the experimental systems, with the transition occurring as the channel widens with $h$ held fixed, so that $w$ decreases and $\alpha$ increases (Hazel et al. 2013).

We believe that a transcritical bifurcation occurs in our model as the obstacle height increases still further, which fundamentally alters the connections between asymmetric and symmetric solution branches for steady propagation modes. A consequence of this transcritical bifurcation is that for higher obstacles, we obtain a stable branch of asymmetric steady propagation modes that exist for all $Q$. At the very lowest flow rates, the stable asymmetric branch is composed of localised fingers, where a narrow finger propagates in the unoccluded region on one side of the obstacle. Hazel et al. (2013) conducted an analysis of the equilibrium finger cross-section in the $(y, z)$ plane far behind the finger tip. In the absence of gravity, their analysis for $Q=0$ shows that symmetric fingers can exist for all obstacle heights, while localised fingers exist only for obstacle heights above a critical value $h_{c}$, which would be $h_{c}=0.17$ for our channel profile. We also obtain a minimum height for localised fingers in the $Q=0$ limit of our depth-averaged model, which gives $h_{c} \equiv h_{\min }=0.21$. The presence of this minimum height in the depth-averaged model is important because it reproduces the experimentally observed behaviour at $Q=0$.

Our model exhibits oscillatory propagation modes with a remarkably similar character to the experimental observations. The oscillations occur at low flow rates for intermediate obstacle heights. For a fixed obstacle height, we find that oscillations in our model disappear at a maximum flow rate through a Hopf bifurcation that we can track directly, but the fate of oscillations at low flow rate is not clear. The oscillations are lost completely for large enough obstacle heights.

The variable channel height profile affects our model through two independent mechanisms. The first of these is a 'viscous mechanism', which is effectively a reduction in mobility along the centre of the channel. We find that this viscous mechanism plays a critical role in determining the bifurcations for steady solutions at low obstacle heights. In other words, the core of the bifurcation structure appears to be a consequence of the localised variations in flow resistance in the channel. It is possible that the entire structure arises because these local variations regularise the degenerate continuum of solutions present in the rectangular channel in the absence of surface tension. The second mechanism is a spatial variation in transverse 
curvature, which we find is necessary to reproduce oscillatory behaviour. A related effect is that the wavelength for oscillations is set by the gradient of the channel height at the smoothed edge of the step, and so is strongly affected by the sharpness parameter chosen. For a sharp step, our model would predict oscillations with infinite wavelength. However, the oscillations observed experimentally have a well-defined wavelength (Pailha et al. 2012), which we expect to be set by three-dimensional effects because the assumption of slow variation in the transverse direction is violated near a sharp step.

Our depth-averaged model neglects a number of factors which may quantitatively affect the results. As mentioned in the discussion of our model derivation, the assumption of a semicircular interface in the transverse direction is simplistic. Park \& Homsy (1984) and Reinelt (1987) proposed corrections valid for low $Q$ to the boundary conditions to account for viscous deformation of the interface and the finite-thickness films deposited above and below the finger. At leading order in small $Q$, their analysis shows that the lateral curvature term in the dynamic boundary condition (2.9) should be multiplied by a factor of $\pi / 4$. This correction can easily be incorporated into our model by a suitable rescaling of $\alpha$ and $Q$, and so has no qualitative effect on the bifurcation structure.

The depth-averaging assumptions will naturally break down when the distance from the channel side-walls is comparable to the channel height. Certain wall effects, such as the no-slip boundary condition, can be obtained in depth-averaged models by use of the Brinkman equations (Nagel et al. 2014), in which a higher-order derivative term is retained in (2.7). Additional complications arise when the finger interface is very close to the channel side-walls. Here drainage effects become important and there can be significant fluid leakage along the corners of the channel cross-section (Wong, Radke \& Morris 1995). Finally, we note that gravitational effects are significant in the millimetre-scale experimental systems investigated by de Lózar et al. (2009), Pailha et al. (2012) and Hazel et al. (2013). However, gravitational effects are much weaker in the micrometre-scale systems investigated by Jisiou et al. (2014), who observed the same range of finger propagation modes discussed in the other experimental papers, including symmetric and asymmetric steadily propagating fingers and the oscillatory propagation modes. By analysing the finger cross-section, Hazel et al. concluded that gravity increases the value of the critical height $h_{c}$ for a given channel but does not qualitatively affect the bifurcation structure. When comparing results for our depth-averaged model with the experimental results, we tend to find that very similar bifurcations occur in the two systems, but with much lower critical obstacle heights in our model than in the experimental results. The direction of this shift in critical height relative to the experimental observations is consistent with gravitational effects; we expect gravity to quantitatively but not qualitatively affect both the propagation modes themselves and the bifurcation structure.

While the experimental investigations for the partially occluded channel concern obstacles which occupy $35-50 \%$ of the channel height, we find that a number of bifurcations occur even for very small obstacles, such as those occupying $5 \%$ of the channel height. These small obstacles may be viewed as perturbations to the Saffman-Taylor system. A number of previous experimental studies have investigated other perturbations to Saffman-Taylor fingering, with a focus on how these perturbations affect the selection problem for low surface tension. The particular perturbations investigated are: a small air bubble just ahead of the finger tip, which is pushed along by the finger as it propagates (Couder et al. 1986a; Couder, Gerard \& Rabaud 1986b; Rabaud et al. 1988); one or more long wires stretched along the 
length of the channel such that the finger interface would intersect these wires (Zocchi et al. 1987; Rabaud et al. 1988); and grooves etched into the plates above and below the finger, each with a maximum depth that is $40 \%$ of the channel height, and aligned parallel to the direction of finger propagation (Rabaud et al. 1988). Each of these perturbations produces localised disturbances of the interface, and indeed all can lead to fingers that are 'anomalous' in that they are narrower than the half-width finger but nonetheless have shapes that agree well with the family found by Saffman \& Taylor. These perturbations can lead to qualitative changes in finger behaviour which have similarities to experimentally observed behaviour for the partially occluded channel.

In wire perturbations, the wire does not intersect the air-fluid interface, but instead a three-dimensional fluid meniscus coats the wire. The finger arranges itself so that its tip is offset from the wire (Zocchi et al. 1987; Rabaud et al. 1988), which can lead to symmetry-breaking behaviour. In theoretical models, the bubble and wire perturbations have been represented either as a finite opening angle (Hong 1989) or as a finite region with lower surface tension on the finger interface that is free to move relative to the tip of the finger (Zocchi et al. 1987). Both these models predict that perturbations can cause narrow fingers and symmetry-breaking behaviour, although quantitative comparison with experiments is difficult.

For each of the experimental perturbations, the finger can also display oscillatory disturbances behind the tip which grow in a direction perpendicular to the propagation direction of the finger (Couder et al. 1986a,b; Rabaud et al. 1988). For bubble perturbations, these disturbances have similarities to the oscillatory propagation modes observed by Pailha et al. (2012) in the partially occluded channel: both have a regular wavelength, and the disturbances are shed a variable distance behind the tip of the finger which is dependent on the finger propagation speed. However, for the oscillatory propagation modes discussed by Pailha et al., there is very little change to the tip of the finger over the period of an oscillation, whereas for bubble perturbations there can be resonance between the bubble and the finger tip, which leads to periodic deformation of the tip shape. Oscillatory disturbances also occur for grooved systems and wire perturbations, but here the observed patterns are much less regular than those arising due to the bubble perturbation. The connections between the solutions induced by the different perturbations remain unclear, but it is conceivable that the overall bifurcation structure is sufficiently generic that any perturbation which serves to regularise the degeneracy at low surface tension will lead to the same types of solution families.

We have studied a system built from some of the most fundamental elements of fluid mechanics: the motion of a viscous fluid bounded by rigid walls and free boundaries, with significant surface-tension effects. For this system, diverse propagation modes and bifurcations have been observed experimentally (de Lózar et al. 2009; Pailha et al. 2012; Hazel et al. 2013), and we have shown here that a depth-averaged model based on the classical model of Saffman \& Taylor is remarkably successful in reproducing the experimentally observed behaviour and explaining the underlying mechanisms. Such depth-averaged models also lead to substantially smaller numerical systems and so give significant freedom in large calculations such as bifurcation tracking or optimisation. Further physical interactions, such as deformation of the channel walls, could be incorporated into the depth-averaged model without destroying its essential simplicity. In this paper, we have been concerned with the effect of non-constant channel height on the propagation of air fingers. The most closely related system is the behaviour of finite-sized bubbles and drops in a similar partially occluded channel, where preliminary experiments 
have shown diverse behaviour, again including symmetry breaking and oscillatory modes (Jisiou et al. 2014). The propagation of air fingers into fluid-filled channels with variable depth is directly applicable to airway reopening problems, and there are interesting questions regarding stabilisation of symmetry-breaking behaviour for these systems. More broadly, microfluidic devices pose a large number of theoretical and practical questions regarding the motion and interactions of bubbles, drops and other deformable bodies in systems where the device geometry is far from simple.

\section{Acknowledgements}

The authors thank Draga Pihler-Puzović and Matthias Heil for many helpful discussions. This work was funded through the EPSRC grant EP/H011579/1.

\section{Appendix A. Linear stability analysis of discretised problem}

For the linear stability analysis, we need to determine the linearised evolution of small perturbations to the interface of a steadily propagating finger. We are already solving for the shape of the propagating finger numerically, so this stability calculation will also require solution of a discretised system. However, there are two equivalent approaches: one is to form separate continuum equations for the base state and the perturbation, and then discretise and solve for each of these separately; the other is to first spatially discretise the continuum system, and then linearise about a steady solution. We choose to use the second approach, but will first discuss the linearised continuum equations for reference.

The linearisation of the depth-averaged equations derived in $\S 2.1$ is mostly straightforward, but as the system is a free boundary problem, evaluation of the boundary conditions is more complicated. In general, we must solve for the pressure field $p$ and the two-dimensional interface position $\boldsymbol{R}$. We let

$$
p(x, y, t)=p_{0}(x, y)+\epsilon \mathrm{e}^{\lambda t} \hat{p}(x, y)
$$

and

$$
\boldsymbol{R}(\xi, t)=\boldsymbol{X}_{0}(\xi)+\epsilon \mathrm{e}^{\lambda t} \hat{\boldsymbol{X}}(\xi), \quad \boldsymbol{X}_{0}=\left(X_{0}, Y_{0}\right), \quad \hat{\boldsymbol{X}}=(\hat{X}, \hat{Y}),
$$

where $\xi$ is an arclength parametrisation of the base state $\boldsymbol{X}_{0}$, with direction such that $\xi$ increases as the finger interface is traversed from the point marked $y_{1}$ to the point marked $y_{2}$ in figure 5 .

The flow is driven by the controlled volume flux imposed ahead of the propagating finger. We therefore keep the dimensionless flow-rate parameter $Q$ constant but allow the tip speed $U$ to vary over the course of an oscillation, so that

$$
\boldsymbol{U}_{F}=\boldsymbol{U}_{0}+\epsilon \mathrm{e}^{\lambda t} \hat{\boldsymbol{U}}, \quad \boldsymbol{U}_{0}=\left(U_{0}, 0\right), \quad \hat{\boldsymbol{U}}=(\hat{U}, 0) .
$$

The kinematic boundary condition (2.8) is expressed in terms of a normal vector $\boldsymbol{n}$, but this need not be a unit normal. We choose $\boldsymbol{n}=\left(Y^{\prime}(\xi),-X^{\prime}(\xi)\right)$, so that

$$
\boldsymbol{n}=\boldsymbol{n}_{0}+\epsilon \mathrm{e}^{\lambda t} \hat{\boldsymbol{n}}, \quad \boldsymbol{n}_{0}=\left(Y_{0}^{\prime},-X_{0}^{\prime}\right), \quad \hat{\boldsymbol{n}}=\left(\hat{Y}^{\prime},-\hat{X}^{\prime}\right) .
$$

We now expand (2.8) in powers of $\epsilon$. At leading order in $\epsilon$, we obtain the kinematic equation for the base state,

$$
\boldsymbol{n}_{0} \cdot \boldsymbol{U}_{0}+b_{0}^{2} \boldsymbol{n}_{0} \cdot \nabla p_{0}=0
$$


where $b_{0} \equiv b\left(Y_{0}\right)$, while at next order in $\epsilon$ we obtain

$$
\begin{aligned}
\boldsymbol{n}_{0} & \cdot \lambda \hat{\boldsymbol{X}}+\hat{\boldsymbol{n}} \cdot\left(\boldsymbol{U}_{0}+b_{0}^{2} \nabla p_{0}\right)+\boldsymbol{n}_{0} \cdot\left(\hat{\boldsymbol{U}}+b_{0}^{2} \nabla \hat{p}+2 \hat{Y} b_{0} b^{\prime}\left(Y_{0}\right) \nabla p_{0}\right) \\
& +b_{0}^{2} \boldsymbol{n}_{0} \cdot(\hat{\boldsymbol{X}} \cdot \nabla) \nabla p_{0}=0 .
\end{aligned}
$$

The dynamic boundary condition (2.9) involves the lateral curvature $\kappa$. If $\xi$ is chosen to increase along the interface from $y_{1}$ to $y_{2}, \kappa$ is defined as

$$
\kappa=\frac{X^{\prime} Y^{\prime \prime}-Y^{\prime} X^{\prime \prime}}{\left(X^{\prime 2}+Y^{\prime 2}\right)^{3 / 2}} .
$$

Upon expanding this expression in powers of $\epsilon$ and using the fact that $\xi$ is a parametrisation of $\boldsymbol{X}_{0}$ with respect to arclength, we find that $\kappa=\kappa_{0}+\epsilon \mathrm{e}^{\lambda t} \hat{\kappa}$, where

$$
\kappa_{0}=X_{0}^{\prime} Y_{0}^{\prime \prime}-Y_{0}^{\prime} X_{0}^{\prime \prime}
$$

and

$$
\hat{\kappa}=-3 \kappa_{0}\left(X_{0}^{\prime} \hat{X}^{\prime}+Y_{0}^{\prime} \hat{Y}^{\prime}\right)+X_{0}^{\prime} \hat{Y}^{\prime \prime}+\hat{X}^{\prime} Y_{0}^{\prime \prime}-Y_{0}^{\prime} \hat{X}^{\prime \prime}-\hat{Y}^{\prime} X_{0}^{\prime \prime} .
$$

With this expression for $\kappa$, we can expand the dynamic boundary condition in powers of $\epsilon$ to obtain

$$
p_{0}-p_{b}+\frac{1}{3 \alpha Q}\left(\frac{1}{b_{0}}+\frac{\kappa_{0}}{\alpha}\right)=0
$$

and

$$
\hat{p}+\hat{\boldsymbol{X}} \cdot \nabla p_{0}+\frac{1}{3 \alpha Q}\left(-\frac{1}{b_{0}^{2}} b^{\prime}\left(Y_{0}\right) \hat{Y}+\frac{\hat{\kappa}}{\alpha}\right)=0 .
$$

In practice, rather than first linearising and then discretising the equations, we analyse linear stability by formulating and solving a generalised eigenvalue problem for the time evolution of linear perturbations to the spatially discretised equations. We use the same finite element discretisation for the spatial problem as for the calculations of steadily propagating fingers and time-dependent evolution.

We write the equations for the spatially discretised system in the general form $\boldsymbol{R}(\dot{\boldsymbol{u}}, \boldsymbol{u})=0$, where the vector $\boldsymbol{u}$ contains all the discretised variables in the problem and $\dot{\boldsymbol{u}}=\partial \boldsymbol{u} / \partial t$. In fact, our time-dependent system (2.7)-(2.12) is linear with respect to the time-derivative terms, and so we may write $\boldsymbol{R}$ as

$$
\boldsymbol{R}=\boldsymbol{M}(\boldsymbol{u}) \dot{\boldsymbol{u}}+\boldsymbol{F}(\boldsymbol{u}),
$$

where $\boldsymbol{M}$ is the mass matrix.

The base state for our linear stability analysis is a steadily propagating finger represented by $\boldsymbol{u}_{0}$, where $\boldsymbol{F}\left(\boldsymbol{u}_{0}\right)=0$. We now consider the time evolution of a small perturbation to this steadily propagating finger, $\boldsymbol{u}=\boldsymbol{u}_{0}+\epsilon \boldsymbol{v}$. For $\epsilon \ll 1$, we find that

$$
\boldsymbol{M}\left(\boldsymbol{u}_{0}\right) \dot{\boldsymbol{v}}+\boldsymbol{J}\left(\boldsymbol{u}_{0}\right) \boldsymbol{v}=0, \quad \boldsymbol{J}=\frac{\partial \boldsymbol{F}}{\partial \boldsymbol{u}}
$$


where $\boldsymbol{J}$ is the same Jacobian matrix as arises when solving for steadily propagating solutions. We decompose $\boldsymbol{v}$ as a sum of eigenvectors $\boldsymbol{v}_{n}$, each of which satisfies $\dot{\boldsymbol{v}}_{n}=$ $\lambda_{n} \boldsymbol{v}_{n}$; thus the eigenvectors are solutions to the generalised eigenvalue problem

$$
(\lambda \boldsymbol{M}+\boldsymbol{J}) \boldsymbol{v}=0 .
$$

The solution $\boldsymbol{u}_{0}$ for steady finger propagation is stable when the real part of $\lambda_{n}$ is negative for all eigenvalues.

For greater efficiency, we prefer to use iterative eigensolvers, which often require a symmetric positive-semidefinite mass matrix. However, our time-dependent system as described in $\S 2.2$ has a non-symmetric mass matrix; to meet the requirements on $\boldsymbol{M}$, we apply a problem-specific linear transformation to (A 14) to yield a suitable transformed mass matrix for the iterative eigensolver.

The only time derivatives in the system (2.7)-(2.12) appear in the equations for pressure at the interface nodes. We now write the vector of unknowns $\boldsymbol{u}$ as $\boldsymbol{u}=$ $(p, x, y, a)^{\mathrm{T}}$, where $p, x$ and $y$ are the pressure and coordinates of the nodes on the finger interface, and $a$ is a vector representing all the other degrees of freedom in the problem. With respect to this vector $\boldsymbol{u}$, the block structure of the mass matrix can be written as

$$
\boldsymbol{M}=\left(\begin{array}{llll}
0 & \boldsymbol{X} & \boldsymbol{Y} & 0 \\
0 & 0 & 0 & 0 \\
0 & 0 & 0 & 0 \\
0 & 0 & 0 & 0
\end{array}\right),
$$

where the sparse square matrices $\boldsymbol{X}$ and $\boldsymbol{Y}$ are the weak forms of $\boldsymbol{e}_{x} \cdot \boldsymbol{n}$ and $\boldsymbol{e}_{y} \cdot \boldsymbol{n}$ integrated around the interface. This mass matrix $\boldsymbol{M}$ is significantly rank-deficient; the rows of $\boldsymbol{M}$ that are entirely zero correspond to algebraic constraints which the perturbation $\boldsymbol{v}$ must always satisfy.

Multiplying (A 14) on the left by an arbitrary square matrix $L$ yields

$$
(\lambda \boldsymbol{L} \boldsymbol{M}+\boldsymbol{L J}) \boldsymbol{v}=0 .
$$

If $\left(\lambda_{n}, \boldsymbol{v}_{n}\right)$ satisfy (A 14), then (A 16) also holds for the same eigenvector-eigenvalue pair. However, the converse statement holds only if $L$ is invertible. We therefore seek a matrix $\boldsymbol{L}$ such that $\boldsymbol{L}$ is invertible and $\boldsymbol{L} \boldsymbol{M}$ is symmetric. If $\boldsymbol{M}$ were invertible, we could satisfy these conditions by taking $\boldsymbol{L}=\boldsymbol{M}^{\mathrm{T}}$. A common solution for rank-deficient $\boldsymbol{M}$ is to take $\boldsymbol{L}=\boldsymbol{J}^{\mathrm{T}}$ and reverse the roles of $\boldsymbol{J}$ and $\boldsymbol{M}$, but this may reduce the sparseness of the generalised eigenproblem. For the mass matrix (A 15), a suitable left multiplier $\boldsymbol{L}$ is given by

$$
\boldsymbol{L}=\left(\begin{array}{cccc}
0 & 0 & \boldsymbol{I}_{1} & 0 \\
\boldsymbol{X}^{\mathrm{T}} & \boldsymbol{Y}^{\mathrm{T}} & 0 & 0 \\
\boldsymbol{Y}^{\mathrm{T}} & -\boldsymbol{X}^{\mathrm{T}} & 0 & 0 \\
0 & 0 & 0 & \boldsymbol{l}_{2}
\end{array}\right)
$$

where $\boldsymbol{I}_{1}$ and $\boldsymbol{l}_{2}$ are appropriately sized identity matrices. The $\boldsymbol{L}$ matrix (A 17) is invertible if $\boldsymbol{X}^{2}+\boldsymbol{Y}^{2}$ is invertible, which holds because the unit normal vector $\boldsymbol{n}$ never vanishes. This choice of $L$ may be easily constructed using the same blocks as $\boldsymbol{M}$, and preserves the sparseness of $\boldsymbol{M}$ and the generalised eigenproblem (A 14).

Finally, we discussed in $\S 2.1$ the need for time-dependent pressure gradients $G_{1,2}$ to avoid convergence problems in time-dependent calculations for oscillatory solutions. 
These scalar variables are determined by conditions on $\mathrm{d} y / \mathrm{d} t$ at the ends of the domain, and so would augment the mass matrix $\boldsymbol{M}$ to

$$
\boldsymbol{M}=\left(\begin{array}{cccccc}
0 & \boldsymbol{X} & \boldsymbol{Y} & 0 & 0 & 0 \\
0 & 0 & 0 & 0 & 0 & 0 \\
0 & 0 & 0 & 0 & 0 & 0 \\
0 & 0 & 0 & 0 & 0 & 0 \\
0 & 0 & \boldsymbol{v}_{1}^{\mathrm{T}} & 0 & 0 & 0 \\
0 & 0 & \boldsymbol{v}_{2}^{\mathrm{T}} & 0 & 0 & 0
\end{array}\right),
$$

where under a suitable reordering, $\boldsymbol{v}_{1}^{\mathrm{T}}=(1,0,0, \ldots)$ and $\boldsymbol{v}_{2}^{\mathrm{T}}=(0,1,0, \ldots)$. We have so far been unable to devise a suitable matrix $\boldsymbol{L}$ for this augmented system. Instead we use the block Krylov-Schur algorithm implemented within the Anasazi solver from Trilinos (Heroux et al. 2003), which does not require a symmetric mass matrix. We find that the boundary condition (2.6) has very little influence on the stability results, and the linear stability results shown in $\S 4$ are for the system with $G_{1,2}$ identically zero.

\section{Appendix B. Consequences of mass conservation}

For steady finger propagation, we must solve for the dimensionless tip speed $U$ as part of the solution. However, a consequence of global mass conservation within the system is that the tip speed and cross-sectional area of the finger far behind the tip are related. For our depth-averaged system, this is equivalent to a relationship between $U$ and $y_{1,2}$ that we can obtain from our equations by integrating (2.7) over the fluid domain and applying the boundary conditions (2.8), (2.10) and (2.12). We thus find that

$$
U=\frac{\int_{-1}^{1} b^{3}(y) \mathrm{d} y}{\int_{y_{1}}^{y_{2}} b(y) \mathrm{d} y}, \quad U_{\min }=\frac{\int_{-1}^{1} b^{3}(y) \mathrm{d} y}{\int_{-1}^{1} b(y) \mathrm{d} y} .
$$

The condition (B 1) does not provide an upper limit for $U$. However, $U$ is bounded below by the minimum value $U_{\min }$, which corresponds to the case where the finger fills the whole width of the channel and $y_{1}=-1, y_{2}=1$. The value of $U_{\min }$ depends on the particular channel profile chosen. We note that the minimum speed is nonzero because we have chosen to non-dimensionalise $U$ against a speed based on the pressure gradient driving the flow.

To aid comparison with the experiments, we further note that $U$ can be related to the 'wet fraction' $m$, where $m$ is defined as the average proportion of fluid that remains in the channel once the finger tip has passed (de Lózar et al. 2009). Fluid initially occupies the whole region $-1<y<1$, but once the finger has passed, or far behind the tip of the finger, fluid occupies only the regions $-1<y<y_{1}$ and $y_{2}<y<1$. The relevant cross-sectional areas can be calculated by integrating the channel height $b(y)$, and so we can write the wet fraction $m$ as

$$
m=1-\frac{\int_{y_{1}}^{y_{2}} b(y) \mathrm{d} y}{\int_{-1}^{1} b(y) \mathrm{d} y}=1-\frac{U_{\min }}{U},
$$

where we have made use of (B 1) to obtain the final expression. 


\section{Appendix C. Solutions at $Q=0$}

When $Q=0$, the pressure field in the fluid has no influence on the interface position, and the dynamic boundary condition (2.9) becomes

$$
\frac{1}{b(y)}+\frac{\kappa}{\alpha}=C,
$$

where $C$ is an arbitrary constant.

The localised fingers that exist at very low $Q$ are typified by finger shapes shown in figure 11. For these localised fingers, the interface curvature in the unoccluded part of the channel is almost constant, but there is a rapid transition near the wall from the constant-curvature region to a straight line adjacent to the wall. There is no corresponding change in channel height near the wall, and so (C1), which describes the limiting case of $Q=0$, cannot capture the transition region. For our $Q=0$ analysis, we apply the simpler boundary condition that the interface makes contact with the wall at a finite value of $x$ and is tangential to the wall at that point. At small but non-zero $Q$, the interface shape must deviate from the solution for $Q=0$ in a small transition region near the channel side-wall in order to match to films with non-zero thickness.

We can make analytical progress by rewriting $(\mathrm{C} 1)$ in the form

$$
\kappa=\frac{\mathrm{d} \psi}{\mathrm{d} \xi}=\alpha\left(C-\frac{1}{b(y)}\right), \quad \frac{\mathrm{d} y}{\mathrm{~d} \xi}=\cos \psi, \quad \frac{\mathrm{d} x}{\mathrm{~d} \xi}=\sin \psi
$$

where $\psi$ is the angle between the outward normal to the air finger and the axial direction $\boldsymbol{e}_{x}$, and $\xi$ is arclength. The equations for $\psi$ and $y$ in (C2) can then be integrated to obtain a relationship between $y$ and $\psi$.

If the finger fills the whole width of the channel, then $\psi=\pi / 2$ at $y=1$ and $\psi=$ $-\pi / 2$ at $y=-1$. Hence

$$
C=\frac{1}{\alpha}+\frac{1}{2} \int_{-1}^{1} \frac{1}{b(y)} \mathrm{d} y,
$$

and the finger shape is symmetric in $y$ if $b(y)$ is symmetric, yielding the symmetric finger shape shown by the dashed line in figure 21 .

When $h=0, b(y)=1$ and the solution is simply $\kappa=1$ everywhere, a semicircular interface. As $h$ increases, the value of $\kappa$ at the midline decreases, and $\kappa$ becomes negative at a finite value of $h$. For large enough $h$, the interface fails to pass over the obstacle; for our channel with $\alpha=10$ and $w=0.25$, we lose the channel-filling solution at $h=h_{\max } \approx 0.41$.

We can calculate the critical height $h_{\max }$ directly for the case where there is no smoothing of the step. The channel height is then piecewise constant, and so the interface shape can be locally constructed from arcs of circles. The two unoccluded regions each have width $1-w$ and $b=b_{1}=1$, and each can be spanned by a single circular arc with radius of curvature $r_{1}$ only if $r_{1} \geqslant(1-w) / 2$. The obstacle has total width $2 w$ and $b=b_{2}=1-h$, and so can be spanned by a single circular arc with radius of curvature $r_{2}$ only if $r_{2} \geqslant w$. In each region, the curvature $\kappa$ is defined by $\kappa_{1,2}= \pm 1 / r_{1,2}$; the choice of sign occurs because the circular arcs may be oriented in either direction. We substitute $b_{1,2}$ and $\kappa_{1,2}$ into (C1) and equate $C$ between the two regions to obtain the condition that

$$
\kappa_{1}-\kappa_{2}=\alpha\left(\frac{1}{b_{2}}-\frac{1}{b_{1}}\right)=\frac{\alpha h}{1-h} .
$$




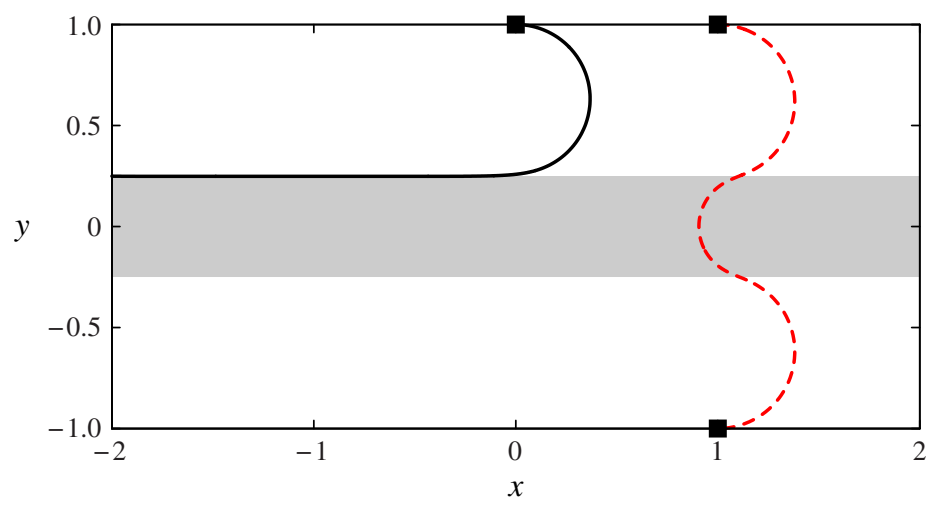

Figure 21. (Colour online) Two solutions at zero $Q$ for $\alpha=10, w=0.25$ and $h=0.4$. The interface touches the wall at a finite value of $x$ and is tangential to the wall there. For this height, the solution may be symmetric, filling the channel (dashed line), or localised to one side of the channel (solid line). For these values of $\alpha$ and $w$, channel-filling solutions are available only for $h \lesssim 0.4$, and localised fingers exist only for $h \gtrsim 0.2$.

The right-hand side of this equation increases with $h$, but $\kappa_{1}-\kappa_{2}$ is constrained due to the conditions on $r_{1,2}$. The maximum value of $\kappa_{1}-\kappa_{2}$ is obtained when $\kappa_{1}=2 /(1-w)$ and $\kappa_{2}=-1 / w$, so we can calculate the maximum height from (C4) as

$$
h_{\max }=\frac{1+w}{1+w+\alpha w(1-w)} .
$$

This result is valid for a sharp channel profile, and yields $h_{\max }=0.40$ when $\alpha=10$ and $w=0.25$.

Returning to the smoothed profile, we can also formulate a boundary value problem for localised fingers, which will satisfy the tangential contact condition at one boundary only (say $y=1$, where $\psi=\pi / 2$ ) and tend towards a line parallel to the channel wall at a finite value of $y=Y$, where $\psi \rightarrow-\pi / 2$ and $\kappa=0$. The condition $\kappa=0$ at $y=Y$ implies that $C=1 / b(Y)$ from (C2), while the requirement that $\psi=-\pi / 2$ becomes an eigenvalue problem for $Y$,

$$
\frac{2}{\alpha}=\frac{1-Y}{b(Y)}-\int_{Y}^{1} \frac{1}{b(y)} \mathrm{d} y .
$$

For a fixed value of $\alpha$, this equation has no solutions for $h<h_{\min }$ and two solutions for $h>h_{\min }$, where $h_{\min }=0.21$ for $\alpha=10$ and $w=0.25$. One of these solutions for $Y$ corresponds to a localised finger, with the finger shape for this solution also shown in figure 21. The second solution to the eigenvalue problem for $Y$ does not correspond to a valid finger shape; the interface instead describes a closed circle in $w<y \leqslant 1$ which never reaches the point $y=Y$.

We can again estimate the minimum height for localised fingers by using a sharp step and a semicircular construction, with $\kappa=2 /(1-w)$ and $b=1$ for $w<y<1$, and with $\kappa=0$ and $b=1 /\left(1-h_{\min }\right)$ on the straight line $y=w$, to find

$$
h_{\min }=\frac{2}{2+\alpha(1-w)},
$$

which yields $h_{\min }=0.21$ for $\alpha=10$ and $w=0.25$. 


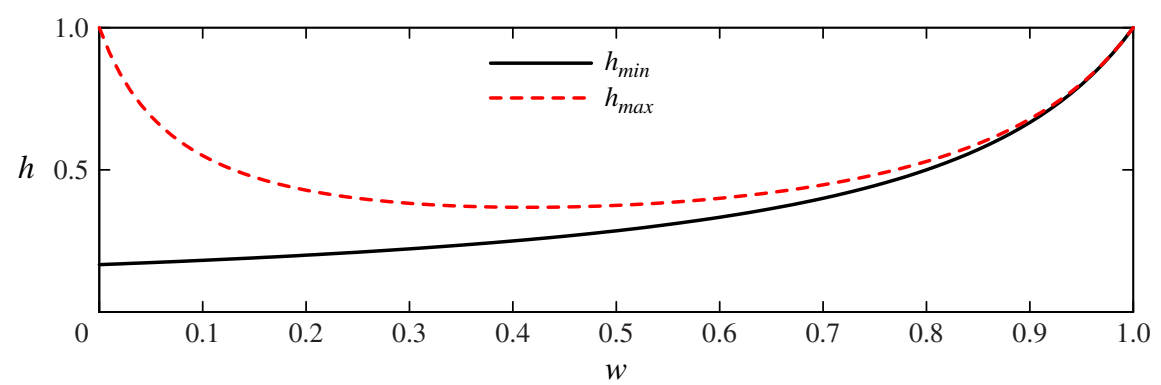

Figure 22. (Colour online) Critical heights for localised and symmetric fingers at $Q=0$. Localised fingers exist above the solid line, and symmetric fingers exist below the dashed line. These critical heights are calculated using the results for the discontinuous step profile $h_{\max }$ and $h_{\min }$ for $\alpha=10$. For all $\alpha>1$ we have verified that the two lines cross at $w=1$ but $h_{\min }<h_{\max }$ elsewhere.

The critical heights $h_{\min }$ and $h_{\max }$ calculated using the sharp-step approximations (C 5) and (C 7) are plotted as $w$ varies in figure 22, with $\alpha$ held fixed. We have verified that with these approximations, $h_{\min }<h_{\max }$ for all $\alpha>1$ with $0<w<1$, and so there is no reordering of the critical heights at $Q=0$.

\section{REFERENCES}

Abbyad, P., Dangla, R., Alexandrou, A. \& Baroud, C. 2011 Rails and anchors: guiding and trapping droplet microreactors in two dimensions. Lab on a Chip 11, 813-821.

Al-Housseiny, T. T., Tsai, P. A. \& Stone, H. A. 2012 Control of interfacial instabilities using flow geometry. Nat. Phys. 8, 747-750.

Casademunt, J. 2004 Viscous fingering as a paradigm of interfacial pattern formation: recent challenges and new results. Chaos 14, 809-824.

Cliffe, K. A., Spence, A. \& TAVener, S. J. 2000 The numerical analysis of bifurcation problems with application to fluid mechanics. Acta Numerica 9, 39-131.

Couder, Y., Cardoso, O., Dupuy, D., Tavernier, P. \& Thom, W. $1986 a$ Dendritic growth in the Saffman-Taylor experiment. Europhys. Lett. 2, 437-443.

Couder, Y., Gerard, N.\& RABAud, M. $1986 b$ Narrow fingers in the Saffman-Taylor instability. Phys. Rev. A 34, 5175-5178.

DAngla, R., LeE, S. \& BARoud, C. N. 2011 Trapping microfluidic drops in wells of surface energy. Phys. Rev. Lett. 107, 124501.

DAwson, G., LEE, S. \& JUEL, A. 2013 The trapping and release of bubbles from a linear pore. J. Fluid Mech. 722, 437-460.

Hazel, A. L., Pailha, M., Cox, S. J. \& Juel, A. 2013 Multiple states of finger propagation in partially occluded tubes. Phys. Fluids 25, 062106.

Heap, A. \& Juel, A. 2008 Anomalous bubble propagation in elastic tubes. Phys. Fluids 20, 081702.

HeIL, M. 1999 Minimal liquid bridges in non-axisymmetrically buckled elastic tubes. J. Fluid Mech. 380, 309-337.

HeIL, M. \& HAZEL, A. L. 2006 oomph-lib: An object-oriented multi-physics finite-element library. In Fluid-Structure Interaction Lecture Notes on Computational Science and Engineering, vol. 53, pp. 19-49. Springer.

Heroux, M., Bartlett, R., Howle, V., Hoekstra, R., Hu, J., Kolda, T., Lehoucq, R., Long, K., Pawlowski, R., Phipps, E., Salinger, A., Thornquist, H., Tuminaro, R., Willenbring, J. \& Williams, A. 2003 An overview of Trilinos. Tech. Rep. SAND2003-2927. Sandia National Laboratories. 
Homsy, G. M. 1987 Viscous fingering in porous media. Annu. Rev. Fluid Mech. 19, 271-311.

Hong, D. C. 1989 Wire perturbations in the Saffman-Taylor problem: pattern selection of asymmetric fingers. Phys. Rev. A 39, 2042-2049.

Jisiou, M., Dawson, G., Thompson, A. B., Mohr, S., Fielden, P. R., Hazel, A. L. \& Juel, A. 2014 Geometry-induced oscillations of finite bubbles in microchannels. Procedia IUTAM 11, 81-88.

Kessler, D. \& Levine, H. 1986 Theory of the Saffman-Taylor 'finger' pattern. II. Phys. Rev. A 33, 2634-2639.

de Lózar, A., Heap, A., Box, F., Hazel, A. L. \& Juel, A. 2009 Tube geometry can force switchlike transitions in the behavior of propagating bubbles. Phys. Fluids 21, 101702.

De Lózar, A., Juel, A. \& HAZel, A. L. 2008 The steady propagation of an air finger into a rectangular tube. J. Fluid Mech. 614, 173-195.

MAXWORTHY, T. 1987 The nonlinear growth of a gravitationally unstable interface in a Hele-Shaw cell. J. Fluid Mech. 177, 207-232.

McCloud, K. V. \& Maher, J. V. 1995 Experimental perturbations to Saffman-Taylor flow. Phys. Rep. 260, 139-185.

Mclean, J. W. \& SAfFman, P. G. 1981 The effect of surface tension on the shape of fingers in a Hele-Shaw cell. J. Fluid Mech. 102, 455-469.

Nagel, M., Brun, P.-T. \& Gallaire, F. 2014 A numerical study of droplet trapping in microfluidic devices. Phys. Fluids 26, 032002.

Pailha, M., Hazel, A. L., Glendinning, P. A. \& Juel, A. 2012 Oscillatory bubbles induced by geometrical constraint. Phys. Fluids 24, 021702.

Park, C.-W. \& Homsy, G. M. 1984 Two-phase displacement in Hele-Shaw cells: theory. J. Fluid Mech. 139, 291-308.

PARK, C. W. \& Homsy, G. M. 1985 The instability of long fingers in Hele-Shaw flows. Phys. Fluids 28, 1583.

Pihler-Puzović, D., Illien, P., Heil, M. \& Juel, A. 2012 Suppression of complex fingerlike patterns at the interface between air and a viscous fluid by elastic membranes. Phys. Rev. Lett. 108, 074502.

Rabaud, M., Couder, Y. \& Gerard, N. 1988 Dynamics and stability of anomalous Saffman-Taylor fingers. Phys. Rev. A 37, 935-947.

REINELT, D. A. 1987 Interface conditions for two-phase displacement in Hele-Shaw cells. J. Fluid Mech. 183, 219-234.

REYNOLDS, O. 1886 On the theory of lubrication and its application to Mr. Beauchamp Tower's experiments, including an experimental determination of the viscosity of olive oil. Phil. Trans. R. Soc. 177, 157-234.

Romero, L. A. 1982 The fingering problem in a Hele-Shaw cell. PhD thesis, California Institute of Technology.

SACKInger, P. A., SChUnK, P. R. \& RAO, R. R. 1996 A Newton-Raphson pseudo-solid domain mapping technique for free and moving boundary problems: a finite element implementation. J. Comput. Phys. 125, 83-103.

SAFFman, P. G. 1986 Viscous fingering in Hele-Shaw cells. J. Fluid Mech. 173, 73-94.

SAFFMAN, P. G. \& TAYLOR, G. I. 1958 The penetration of a fluid into a porous medium or Hele-Shaw cell containing a more viscous liquid. Proc. R. Soc. Lond. A 245, 312-329.

Tabeling, P., Zocchi, G. \& Libchaber, A. 1987 An experimental study of the Saffman-Taylor instability. J. Fluid Mech. 177, 67-82.

Tanveer, S. 1987 Analytic theory for the linear stability of the Saffman-Taylor finger. Phys. Fluids 30, 2318-2329.

TANVEeR, S. 2000 Surprises in viscous fingering. J. Fluid Mech. 409, 273-308.

TAYlor, G. \& SAFfman, P. G. 1959 A note on the motion of bubbles in a Hele-Shaw cell and porous medium. Q. J. Mech. Appl. Maths 12, 265-277. 
Vanden-Broeck, J. -M. 1983 Fingers in a Hele-Shaw cell with surface tension. Phys. Fluids 26, 2033-2034.

Wong, H., RADKe, C. J. \& MorRIS, S. 1995 The motion of long bubbles in polygonal capillaries. Part 1. Thin films. J. Fluid Mech. 292, 71-94.

ZIENKIEWICZ, O. C. \& ZHU, J. Z. 1992 The superconvergent patch recovery and a posteriori error estimates. Part 1: the recovery technique. Intl J. Numer. Meth. Engng 33, 1331-1364.

Zocchi, G., Shaw, B. E., Libchaber, A. \& Kadanoff, L. P. 1987 Finger narrowing under local perturbations in the Saffman-Taylor problem. Phys. Rev. A 36 (4), 1894-1900. 